\title{
Some probabilistic trees with algebraic roots
}

\author{
Olivier Bernardi * \\ Department of Mathematics \\ Brandeis University \\ Waltham, MA, USA \\ bernardi@brandeis.edu
}

\author{
Alejandro H. Morales ${ }^{\dagger}$ \\ Department of Mathematics \\ UCLA \\ Los Angeles, CA, USA \\ ahmorales@math.ucla.edu
}

Submitted: Jan 4, 2015; Accepted: May 5, 2016; Published: May 13, 2016

Mathematics Subject Classifications: 05A15, 05C30, 60C05

\begin{abstract}
We consider several probabilistic processes defining a random graph. One of these processes appeared recently in connection with a factorization problem in the symmetric group. For each process, we prove that the probability for the random graph to be a tree has an unexpectedly simple expression, which is independent of most parameters of the problem. This raises several open questions.
\end{abstract}

Keywords: branched polymers, random graphs, random trees, matrixtree theorem

\section{Introduction: an example}

In this paper we consider several probabilistic processes defining a random graph. These processes were originally motivated by factorization problems in the symmetric group investigated in [1]. Our main result is a formula, for each of the probabilistic processes, of the probability that the random graph is a tree. This probability formula turns out to be surprisingly simple, and independent of most parameters of the processes. This is reminiscent of the result of Kenyon and Winkler [3] about 2-dimensional branched polymers. We conjecture that more results of this type should hold, but we were not able to prove them.

Before describing our results in full details, let us describe one particular case. Let $k$ be a positive integer. Given a tuple $\mathbf{S}=\left(S_{1}, \ldots, S_{k-1}\right)$ of $k-1$ proper subsets of $[k]:=\{1,2, \ldots, k\}$, we define $G(\mathbf{S})$ as the digraph having vertex set $[k]$ and arc set $\left\{a_{1}, \ldots, a_{k-1}\right\}$, where the arc $a_{i}$ has origin $i$ and endpoint the unique integer $j$ in $[k] \backslash S_{i}$

*Supported by NSF grants DMS-1308441, DMS-1400859, and ERC ExploreMaps.

†'Supported by a CRM-ISM postdoctoral fellowship. 
such that $\{i+1, i+2, \ldots, j-1\} \subseteq S_{i}$, with the integers considered cyclically modulo $k$. For instance, if $k=7$ and $S_{5}=\{1,3,6,7\}$, then the arc $a_{5}$ has origin 5 and endpoint 2 . In Figure 1 we have drawn some digraphs $G(\mathbf{S})$ in the case $k=3$. We now fix a tuple $\mathbf{p}=\left(p_{1}, \ldots, p_{k}\right)$ of non-negative integers, and choose uniformly at random a tuple $\mathbf{S}=$ $\left(S_{1}, \ldots, S_{k-1}\right)$ of $k-1$ proper subsets of $[k]$ such that for all $j \in[k]$ the integer $j$ is contained in exactly $p_{j}$ of the subsets $S_{1}, \ldots, S_{k-1}$. This gives a random digraph $G(\mathbf{S})$. One of the results proved in this paper is that the probability that $G(\mathbf{S})$ is a tree (oriented toward the vertex $k$ ) is equal to $1-p_{k} /(k-1)$. This result is unexpectedly simple, especially because it does not depend on the parameters $p_{1}, \ldots, p_{k-1}$.

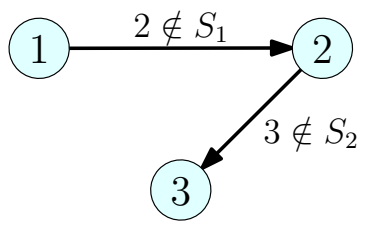

(a)

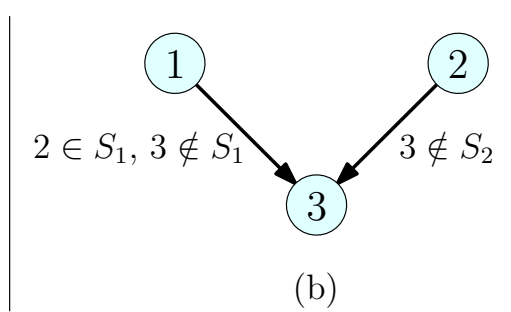

(b)

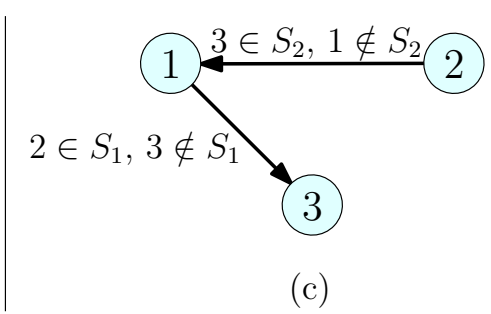

(c)

Figure 1: We represent above the three situations for which the digraph $G(\mathbf{S})$ is a tree, with $k=3$ and $\mathbf{S}=\left(S_{1}, S_{2}\right)$.

Let us investigate in more detail the case $k=3$ of the aforementioned result. The situation is represented in Figure 1. By definition, the tuple $\mathbf{S}=\left(S_{1}, S_{2}\right)$ is a pair of proper subsets of $\{1,2,3\}$, and there are three possible trees with vertex set $\{1,2,3\}$ (in general, there are $k^{k-2}$ possible Cayley trees). Let $A, B$, and $C$ be respectively the events leading to the trees represented in parts (a), (b), and (c) of Figure 1. For instance,

$$
C=\left\{2 \in S_{1}\right\} \cap\left\{3 \notin S_{1}\right\} \cap\left\{3 \in S_{2}\right\} \cap\left\{1 \notin S_{2}\right\} .
$$

Now it is not hard to check that the event $C$ has the same probability as the event

$$
C^{\prime}=\left\{2 \in S_{1}\right\} \cap\left\{3 \notin S_{2}\right\} \cap\left\{3 \in S_{1}\right\} \cap\left\{1 \notin S_{1}\right\} .
$$

Moreover the events $A, B$ and $C^{\prime}$ are disjoint and their union is

$A \cup B \cup C^{\prime}=\left(\left\{2 \notin S_{1}\right\} \cup\left\{2 \in S_{1} \cap 3 \notin S_{1}\right\} \cup\left\{2 \in S_{1} \cap 3 \in S_{1} \cap 1 \notin S_{1}\right\}\right) \cap\left\{3 \notin S_{2}\right\}$.

But since $S_{1}$ is by definition a proper subset of $\{1,2,3\}$, the first condition in the above clause is always satisfied. It follows that

$$
\mathbb{P}(A \cup B \cup C)=\mathbb{P}\left(A \cup B \cup C^{\prime}\right)=\mathbb{P}\left(3 \notin S_{2}\right)=1-p_{3} / 2,
$$

as claimed. Observe that the individual probabilities of the trees represented in Figure 1 $d o$ depend on the value of $p_{1}$ and $p_{2}$, but the sum of these probabilities is independent of $p_{1}$ and $p_{2}$. 
The rest of the paper is organized as follows. In Section 2, we state the main results of the paper. In Section 3, we express the probability that our random graphs are trees as the "probability of a determinant of a matrix of events". In Section 4, we compute the probability of these determinants. This computation is rather technical, and uses some sign reversing involutions. We conclude in Section 5 with some conjectures about another random process, and some open questions.

\section{Main results}

In this section we fix some notation and state our main results. We denote by $|A|$ the cardinality of a set $A$. We denote $A \uplus B$ the disjoint union of two sets $A, B$. For a positive integer $k$, we denote by $[k]$ the set of integers $\{1,2, \ldots, k\}$. For $i, j \in[k]$, we denote by ]$i, j]$ the set of integers $\{i+1, i+2, \ldots, j\}$, where integers are considered cyclically modulo $k$. For instance $] i, i]=\emptyset,] i, i+1]=\{i+1\}$, and $] i, i-1]=[k] \backslash\{i\}$. For an integer $r$ and a tuple $\mathbf{p}=\left(p_{1}, \ldots, p_{k}\right)$ of non-negative integers, we denote by $\mathcal{S}_{\mathbf{p}, r}$ the set of tuples $\left(S_{1}, \ldots, S_{r}\right)$ such that for all $i \in[r], S_{i}$ is a subset of $[k]$ and for all $j \in[k]$ the integer $j$ is contained in exactly $p_{j}$ of the subsets $S_{1}, \ldots, S_{r}$. We also denote by $\mathcal{R}_{\mathbf{p}, r}$ the set of tuples $\left(S_{1}, \ldots, S_{r}\right) \in \mathcal{S}_{\mathbf{p}, r}$ such that $S_{i} \neq[k]$ for all $i \in[r]$.

We now define three ways of associating a digraph to a an element in $\mathcal{S}_{\mathbf{p}, r}$, using three mappings $\alpha, \beta, \gamma$. The mappings $\alpha, \beta, \gamma$ take as argument an integer $i \in[k]$ and a subset $S \subseteq[k]$ and are defined as follows:

- $\alpha(i, S)=i$ if $S=[k]$, and otherwise $\alpha(i, S)$ is the integer $j \in[k]$ such that $j \notin S$ but $] i, j-1] \subseteq S$.

- $\beta(i, S)=i$ if $S=[k]$, and otherwise $\beta(i, S)$ is the integer $j \in[k]$ such that $j+1 \notin S$ but $] i, j] \subseteq S$.

- $\gamma(i, S)=i$ if $S=[k], \gamma(i, S)=i-1$ if $i \in S$, and otherwise $\gamma(i, S)$ is the integer $j \in[k]$ such that $j+1 \notin S$ but $] i, j] \subseteq S$.

The mappings $\alpha, \beta, \gamma$ are represented in Figure 2 .

We now use the mappings $\alpha, \beta, \gamma$ to define digraphs. Let $\mathbf{S}=\left(S_{1}, \ldots, S_{r}\right) \in \mathcal{S}_{\mathbf{p}, r}$, and let $f$ be a surjection from $[k-1]$ to $[r]$. For $\zeta \in\{\alpha, \beta, \gamma\}$, we define $G_{\zeta}(\mathbf{S}, f)$ to be the digraph with vertex set $[k]$ and $\operatorname{arc}$ set $A=\left\{a_{1}, \ldots, a_{k-1}\right\}$ where $a_{i}=\left(i, \zeta\left(i, S_{f(i)}\right)\right)$ for all $i \in[k-1]$. For instance, the digraph $G(\mathbf{S})$ defined in Section 1 corresponds to the case $r=k-1$ and $G(\mathbf{S})=G_{\alpha}(\mathbf{S}, \mathrm{Id})$ where Id is the identity mapping from $[k-1]$ to $[k-1]$. Observe that the graph $G_{\zeta}(\mathbf{S}, f)$ has loops unless $\mathbf{S} \in \mathcal{R}_{\mathbf{p}, r}$.

We are now interested in the probability that the digraph $G_{\zeta}(\mathbf{S}, f)$ is a tree. Observe that in this case the tree is oriented toward the vertex $k$ (since every vertex in $[k-1]$ has one outgoing arc). Our main result is the following.

Theorem 1. Let $k$ and $r$ be positive integers such that $r<k$, and let $\boldsymbol{p}=\left(p_{1}, \ldots, p_{k}\right)$ be a tuple of non-negative integers. Let $\boldsymbol{S}=\left(S_{1}, \ldots, S_{r}\right)$ be a uniformly random element of $\mathcal{R}_{p, r}$ (supposing that this set is non-empty), and let $f$ be a uniformly random surjection 


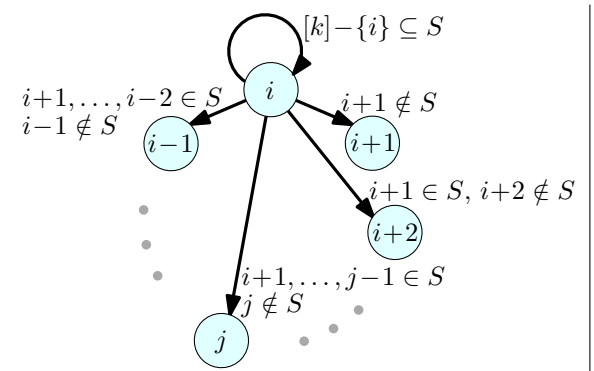

$(\alpha)$ Rule " $i+1, \ldots, j-1, \bar{j}$ ".

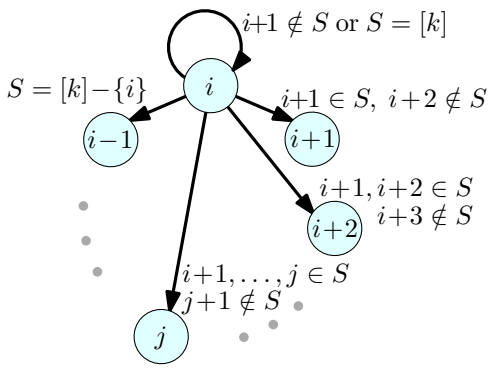

( $\beta$ ) Rule " $i+1, \ldots, j, \overline{j+1}$ ".

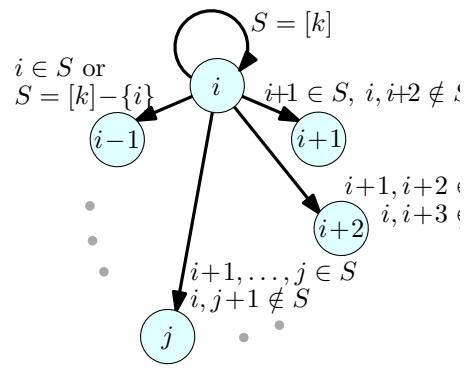

$(\gamma)$ Rule " $\bar{i}, i+1, \ldots, j, \overline{j+1}$ '

Figure 2: Rules $\alpha, \beta, \gamma$ for creating an arc of the complete graph $K_{k}$ (an informal description of the rule is given between quotation marks where $\bar{i}$ means that $i \notin S$ ).

from $[k-1]$ to $[r]$ independent from $\boldsymbol{S}$. For $\zeta \in\{\alpha, \beta, \gamma\}$, let $P_{\zeta}(\boldsymbol{p}, r)$ be the probability that the random digraph $G_{\zeta}(\boldsymbol{S}, f)$ is a tree. Then

(a) $P_{\alpha}(\boldsymbol{p}, r)=1-p_{k} / r$. This is equal to the probability that $k \notin S_{1}$.

(b) $P_{\beta}(\boldsymbol{p}, r)=\left|\mathcal{R}_{\boldsymbol{q}, r-1}\right| /\left|\mathcal{R}_{\boldsymbol{p}, r}\right|$ where $\boldsymbol{q}=\left(p_{1}, p_{2}-1, p_{3}-1, \ldots, p_{k}-1\right)$. This is equal to the probability that $S_{1}=\{2,3, \ldots, k\}$.

(c) $P_{\gamma}(\boldsymbol{p}, r)=\sum_{j=1}^{k}\left|\mathcal{R}_{\boldsymbol{q}^{(j)}, r-1}\right| /\left|\mathcal{R}_{\boldsymbol{p}, r}\right|$, where $\boldsymbol{q}^{(j)}=\left(q_{1}^{(j)}, \ldots, q_{k}^{(j)}\right)$ and for all $i, j \in[k]$, $q_{i}^{(j)}=p_{i}-1$ if $i \neq j$ and $q_{j}^{(j)}=p_{j}$. This is equal to the probability that $\left|S_{1}\right|=k-1$.

Observe that the cardinality of $\mathcal{R}_{\mathbf{p}, r}$ appearing in Theorem 1 can be expressed as the coefficient of $x_{1}^{p_{1}} x_{2}^{p_{2}} \cdots x_{k}^{p_{k}}$ in the polynomial $\left(\prod_{i=1}^{k}\left(x_{i}+1\right)-\prod_{i=1}^{k} x_{i}\right)^{r}$. The case (c) of Theorem 1 was needed to complete the combinatorial proof described in [1] of Jackson's formula [2]. Before embarking on the proof of Theorem 1, we make a few remarks.

Remark 2. Theorem 1 can be stated in terms of uniformly random tuples $\mathbf{S}$ in $\mathcal{S}_{\mathbf{p}, r}$ instead of in $\mathcal{R}_{\mathbf{p}, r}$. More precisely, for $\zeta \in\{\alpha, \beta, \gamma\}$, if $\mathbf{S}$ is a uniformly random tuple in $\mathcal{S}_{\mathbf{p}, r}$, and $f$ is a uniformly random surjection from $[k-1]$ to $[r]$ independent from $\mathbf{S}$, then the probability for the graph $G_{\zeta}(\mathbf{S}, f)$ to be a tree is $\frac{\left|\mathcal{R}_{\mathbf{p}, r}\right|}{\left|\mathcal{S}_{\mathbf{p}, r}\right|} P_{\zeta}(\mathbf{p}, r)$. This is simply because the graph $G_{\zeta}(\mathbf{S}, f)$ is never a tree if $\mathbf{S} \in \mathcal{S}_{\mathbf{p}, r} \backslash \mathcal{R}_{\mathbf{p}, r}$.

Remark 3. In the case $r=k-1$, the result of Theorem 1 , can be stated without referring to a random surjection $f$. Indeed, for $\mathbf{S} \in \mathcal{R}_{\mathbf{p}, r}$ and $\zeta \in\{\alpha, \beta, \gamma\}$, let us define $G_{\zeta}(\mathbf{S})$ as the graph $G_{\zeta}(\mathbf{S}, \mathrm{Id})$ where $\mathrm{Id}$ is the identity mapping from $[k-1]$ to $[k-1]$. Then the probability $P_{\zeta}(\mathbf{p}, k-1)$ that the graph $G_{\zeta}(\mathbf{S})$ is a tree has the same expression as in Theorem 1. For instance, the probability that $G_{\alpha}(\mathbf{S})$ is a tree is $1-p_{k} /(k-1)$, as claimed in Section 1. Indeed, in the particular case $r=k-1$ of Theorem 1 , the surjection $f$ would be a bijection from $[k-1]$ to $[k-1]$ independent from $\mathbf{S}$. But then the tuple 
$\left(S_{f(1)}, \ldots, S_{f(k-1)}\right)$ has the same distribution as $\mathbf{S}=\left(S_{1}, \ldots, S_{k-1}\right)$, hence the surjection $f$ does not affect probabilities.

Remark 4. The results in Theorem 1 would hold for any probability distribution on the tuples $\mathbf{S}=\left(S_{1}, \ldots, S_{k}\right)$ of proper subsets of $[k]$, provided that the probability of a tuple only depends on the total number of occurrences of each integer $i \in[k]$. For instance, the probability that $G_{\alpha}(\mathbf{S}, f)$ is a tree would still be equal to the probability that $k \notin S_{1}$ for such a probability distribution. This result follows from Theorem 1 since one can always condition on the total number of occurrences of each integer $i \in[k]$.

Remark 5. A slightly weaker version of Theorem 1 can be obtained by not requiring the function $f$ to be surjective. More precisely, for $\zeta \in\{\alpha, \beta, \gamma\}$ and for any positive integer $r$, if $\mathbf{S}$ is a uniformly random tuple in $\mathcal{R}_{\mathbf{p}, r}$, and $f$ is a uniformly random function from $[k-1]$ to $[r]$ independent from $\mathbf{S}$, then the probability $P_{\zeta}(\mathbf{p}, r)$ that $G_{\zeta}(\mathbf{S}, f)$ has the same expression as in Theorem 1. For instance, the probability that $G_{\alpha}(\mathbf{S}, f)$ is a tree is $1-p_{k} / r$. Indeed, this result follows from Theorem 1 by conditioning on the cardinality of the image $\operatorname{Im}(f)$ of the function $f$, and by the number of occurrences of each integer $i \in[k]$ in the subsets $\left(S_{j}\right)_{j \in \operatorname{Im}(f)}$. It is actually this version of Theorem 1 (in the case $\zeta=\gamma)$ which was needed in [1].

\section{Determinantal expressions for the probabilities $\boldsymbol{P}_{\zeta}(\mathrm{p}, \boldsymbol{r})$.}

In this section, we express $P_{\alpha}(\mathbf{p}, r), P_{\beta}(\mathbf{p}, r), P_{\gamma}(\mathbf{p}, r)$ as the probabilities of the determinants of some matrices of events. This is done by first applying the matrix-tree theorem, and then simplifying the Laplacian matrices.

\subsection{Matrix-tree theorem over the ring of events}

We first recall the matrix-tree theorem (in its weighted, directed version). Let $K_{n}$ denote the complete digraph having vertex set $[n]$ and arc set $A=\{(i, j), i \neq j\}$. We call spanning tree of $K_{n}$ rooted at $n$ a set of arcs $T \subseteq A$ not containing any cycle and such that every vertex $i \in[n-1]$ is incident to exactly one outgoing $\operatorname{arc}$ in $T$ (this is equivalent to asking that $T$ is a spanning tree of $K_{n}$ "oriented toward" the root vertex $n$ ). We denote by $\mathcal{T}_{n}$ the set of spanning trees of $K_{n}$ rooted at $n$.

Let $\mathcal{R}=(R,+, \otimes)$ be a commutative ring. Recall that the determinant of a matrix $M=\left(M_{i, j}\right)_{i, j \in[k]}$ with coefficients in $R$ is

$$
\operatorname{det}(M)=\sum_{\pi \in \mathfrak{S}_{k}} \epsilon(\pi) \bigotimes_{i \in[k]} M_{i, \pi(i)}
$$

where $\mathfrak{S}_{k}$ is the set of permutations of $[k]$, and $\epsilon(\pi)$ is the sign of the permutation $\pi$. The matrix-tree theorem states that for any weights $w(i, j) \in R$ associated to the arcs $(i, j)$ of $K_{n}$,

$$
\sum_{T \in \mathcal{T}_{n}} \bigotimes_{(i, j) \in T} w(i, j)=\operatorname{det}(L)
$$


where $L=\left(L_{i, j}\right)_{i, j \in[n-1]}$ is the reduced Laplacian matrix, defined by $L_{i, j}=-w(i, j)$ if $i \neq j$ and $L_{i, i}=\sum_{j \in[n] \backslash\{i\}} w(i, j)$. For a proof of (1), see for instance the combinatorial proof of the matrix-tree theorem given in [4].

Remark 6. Observe that (1) gives results about the spanning trees of any digraph $G$ with vertex set $[n]$, because one can restrict one's attention to the spanning trees of $G$ simply by setting $w(i, j)=0$ for all arcs $(i, j)$ not in $G$. Observe also that the weights $w(n, j)$ are actually irrelevant in (1).

Next we define a ring of events. Let $(\Omega, \mathcal{A}, \mathbb{P})$ be a probability space, where $\Omega$ is the sample set, $\mathcal{A}$ is the set of events (which is a $\sigma$-algebra on $\Omega$ ), and $\mathbb{P}$ is the probability measure. In our applications, $\Omega$ will be a finite set and $\mathcal{A}$ will be the powerset $\mathcal{P}(\Omega)$. Let $\mathbb{C}[\mathcal{A}]$ be the set of (formal) finite linear combinations of events, with coefficients in the field $\mathbb{C}$ (i.e., the free $\mathbb{C}$-module with basis $\mathcal{A}$ ). The elements of $\mathbb{C}[\mathcal{A}]$ are called generalized events and are of the form $\lambda_{1} A_{1}+\cdots+\lambda_{s} A_{s}$ with $\lambda_{1}, \ldots, \lambda_{s} \in \mathbb{C}$ and $A_{1}, \ldots, A_{s} \in \mathcal{A}$. We then consider the commutative ring $\mathcal{R}=(\mathbb{C}[\mathcal{A}],+, \cap)$. Here the intersection operation " $\cap$ " is defined to act distributively on $\mathbb{C}[\mathcal{A}]$, that is, we define

$$
\left(\sum_{i=1}^{s} \lambda_{i} A_{i}\right) \cap\left(\sum_{j=1}^{t} \lambda_{j}^{\prime} A_{j}^{\prime}\right):=\sum_{i=1}^{s} \sum_{j=1}^{t} \lambda_{i} \lambda_{j}^{\prime} A_{i} \cap A_{j}^{\prime} .
$$

We now extend the probability measure $\mathbb{P}$ from $\mathcal{A}$ to $\mathbb{C}[\mathcal{A}]$ by linearity. More concretely, we set

$$
\mathbb{P}\left(\lambda_{1} A_{1}+\cdots+\lambda_{s} A_{s}\right)=\lambda_{1} \mathbb{P}\left(A_{1}\right)+\cdots+\lambda_{s} \mathbb{P}\left(A_{s}\right) .
$$

This makes $\mathbb{P}$ a linear map from $\mathbb{C}[\mathcal{A}]$ to $\mathbb{R}$. We define the $\mathbb{P}$-determinant of a matrix of generalized events $M=\left(M_{i, j}\right)_{i, j \in[k]}$ as $\mathbb{P} \operatorname{det}(M):=\mathbb{P}(\operatorname{det}(M))$. By definition,

$$
\mathbb{P} \operatorname{det}(M)=\sum_{\pi \in \mathfrak{S}_{k}} \epsilon(\pi) \mathbb{P}\left(\bigcap_{i \in[k]} M_{i, \pi(i)}\right) .
$$

For a matrix $E=\left(E_{i, j}\right)_{i \in[n-1], j \in[n]}$ of generalized events $E_{i, j} \in \mathbb{C}[\mathcal{A}]$, we define its reduced Laplacian matrix $L=\left(L_{i, j}\right)_{i, j \in[n-1]}$ by setting for all $i \in[n-1] L_{i, j}=-E_{i, j}$ if $i \neq j$ and $L_{i, i}=\sum_{j \neq i} E_{i, j}$. We now state an immediate consequence of (1).

Lemma 7. Let $E=\left(E_{i, j}\right)_{i \in[n-1], j \in[n]}$ be a matrix of generalized events and let $L=$ $\left(L_{i, j}\right)_{i, j \in[n-1]}$ be its reduced Laplacian matrix. Then

$$
\sum_{T \in \mathcal{T}_{n}} \mathbb{P}\left(\bigcap_{(i, j) \in T} E_{i, j}\right)=\mathbb{P} \operatorname{det}(L)
$$

Proof. Applying (1) over the $\operatorname{ring} \mathcal{R}=(\mathbb{C}[\mathcal{A}],+, \cap)$ with weight $w(i, j)=E_{i, j}$ gives

$$
\sum_{T \in \mathcal{T}_{n}} \bigcap_{(i, j) \in T} E_{i, j}=\sum_{\pi \in \mathfrak{S}_{n-1}} \epsilon(\pi) \bigcap_{i \in[n-1]} L_{i, \pi(i)} .
$$

Applying the linear map $\mathbb{P}$ to both sides of the above identity gives the Lemma. 
Before closing this section we define an equivalence relation on the set $\mathbb{C}[A]$ of generalized events. Let $A_{1}, \ldots, A_{m}, B_{1}, \ldots, B_{n}$ be events in $\mathcal{A}$, and let $\lambda_{1}, \ldots, \lambda_{m}, \nu_{1} \ldots, \nu_{n}$ be complex numbers. We say that the generalized event $A=\sum_{i=1}^{m} \lambda_{i} A_{i}$ and $B=$ $\sum_{i=1}^{n} \nu_{i} B_{i}$ are equivalent, and we denote this $A \sim B$, if for all $\omega \in \Omega$, the quantities $A_{\omega}=\sum_{i=1}^{m} \lambda_{i} \mathbf{1}_{\omega \in A_{i}}$ and $B_{\omega}=\sum_{i=1}^{n} \nu_{i} \mathbf{1}_{\omega \in B_{i}}$ are equal. For instance, for all $E, F \in \mathcal{A}$, the generalized events $E \cup F$ and $E+F-E \cap F$ are equivalent. Also the event $\emptyset$ and the generalized event 0 are equivalent. It is easy to see that $\sim$ is an equivalence relation (symmetric, reflexive, transitive) and that if $E \sim F$ then $\mathbb{P}(E)=\mathbb{P}(F)$. Moreover, if $E \sim F$ and $E^{\prime} \sim F^{\prime}$ then $\lambda \cdot E \sim \lambda \cdot F, E+F \sim E^{\prime}+F^{\prime}$ and $E \cap F \sim E^{\prime} \cap F^{\prime}$. We say that two matrices of generalized events $E=\left(E_{i, j}\right)_{i, j \in[n]}$ and $F=\left(F_{i, j}\right)_{i, j \in[n]}$ are equivalent if $E_{i, j} \sim F_{i, j}$ for all $i \in[n], j \in[n]$. The preceding properties immediately imply the following result.

Lemma 8. If $E$ and $F$ are equivalent matrices of events, then they have the same $\mathbb{P}$ determinant.

\section{2 $P_{\zeta}(\mathrm{p}, r)$ as a $\mathbb{P}$-determinant}

We fix $r, k$, and $\mathbf{p}$ as in Theorem 1 . We define a probability space $(\Omega, \mathcal{A}, \mathbb{P})$ in the following way:

- $\Omega$ is the set of pairs $(\mathbf{S}, f)$, where $\mathbf{S}$ is in $\mathcal{S}_{\mathbf{p}, r}$ and $f$ is a surjection from $[k-1]$ to $[r]$.

- $\mathcal{A}$ is the power set $\mathcal{P}(\Omega)$,

- $\mathbb{P}$ is the uniform distribution on $\Omega$.

We denote by $\Omega_{n}$ the set of triples $(\mathbf{S}, f, \pi)$, where $(\mathbf{S}, f)$ is in $\Omega$ and $\pi$ is a permutation of $[n]$. For a matrix of events $E=\left(E_{i, j}\right)_{i, j \in[n]}$ we denote by $\Omega(E)$ the set of triples $(\mathbf{S}, f, \pi) \in \Omega_{n}$ such that $(\mathbf{S}, f)$ is in the intersection $\bigcap_{i=1}^{n} E_{i, \pi(i)}$. Observe that

$$
\operatorname{Pdet}(E)=\frac{1}{|\Omega|} \sum_{(\mathbf{S}, f, \pi) \in \Omega(E)} \epsilon(\pi) .
$$

We now express the probabilities $P_{\zeta}(\mathbf{p}, r)$ defined in Theorem 1 as $\mathbb{P}$-determinants. By definition, for $\zeta \in\{\alpha, \beta, \gamma\}, P_{\zeta}(\mathbf{p}, r)$ is the conditional probability, in the space $(\Omega, \mathcal{A}, \mathbb{P})$, that the random digraph $G_{\zeta}(\mathbf{S}, f)$ is a tree given that $\mathbf{S}=\left(S_{1}, \ldots, S_{r}\right)$ is in $\mathcal{R}_{\mathbf{p}, r}$ (equivalently, given that none of the subsets $S_{1}, \ldots, S_{r}$ is equal to $\left.[k]\right)$. Since the random digraph $G_{\zeta}(\mathbf{S}, f)$ is never a tree unless $\mathbf{S}$ is in $\mathcal{R}_{\mathbf{p}, r}$ (because $G_{\zeta}(\mathbf{S}, f)$ has loops if $\mathbf{S} \notin \mathcal{R}_{\mathbf{p}, r}$ ) one gets

$$
P_{\zeta}(\mathbf{p}, r)=\frac{\mathbb{P}\left(G_{\zeta}(\mathbf{S}, f) \text { is a tree }\right)}{\mathbb{P}\left(\mathbf{S} \in \mathcal{R}_{\mathbf{p}, r}\right)}=\frac{\left|\mathcal{S}_{\mathbf{p}, r}\right|}{\left|\mathcal{R}_{\mathbf{p}, r}\right|} \sum_{T \in \mathcal{T}_{k}} \mathbb{P}\left(G_{\zeta}(\mathbf{S}, f)=T\right),
$$

where $\mathcal{T}_{k}$ is the set of spanning trees of $K_{k}$ rooted at $k$.

We now rewrite (3) in a form suitable to apply Lemma 7 . For $\zeta$ in $\{\alpha, \beta, \gamma\}, i \in[k-1]$, and $j \in[k]$, we define the event $E_{\zeta, i, j} \subseteq \Omega$ as the set of pairs $(\mathbf{S}, f)$ in $\Omega$ such that 
$\zeta\left(i, S_{f(i)}\right)=j$. In other words, $E_{\zeta, i, j}$ is the event "the arc $a_{i}$ of the digraph $G_{\zeta}(\mathbf{S}, f)$ is $(i, j)$ ". By definition, for any tree $T$ in $\mathcal{T}_{k}$, the event $G_{\zeta}(\mathbf{S}, f)=T$ is equal to $\bigcap_{(i, j) \in T} E_{\zeta, i, j}$. Thus,

$$
P_{\zeta}(\mathbf{p}, r)=\frac{\left|\mathcal{S}_{\mathbf{p}, r}\right|}{\left|\mathcal{R}_{\mathbf{p}, r}\right|} \sum_{T \in \mathcal{T}_{k}} \mathbb{P}\left(\bigcap_{(i, j) \in T} E_{\zeta, i, j}\right) .
$$

Hence by Lemma 7,

$$
P_{\zeta}(\mathbf{p}, r)=\frac{\left|\mathcal{S}_{\mathbf{p}, r}\right|}{\left|\mathcal{R}_{\mathbf{p}, r}\right|} \mathbb{P} \operatorname{det}\left(L_{\zeta}\right)
$$

where $L_{\zeta}=\left(L_{\zeta, i, j}\right)_{i, j \in[k-1]}$ is the reduced Laplacian matrix of $E_{\zeta}=\left(E_{\zeta, i, j}\right)_{i \in[k-1], j \in[k]}$.

Next, we define a matrix $L_{\zeta}^{\prime}$ equivalent to the reduced Laplacian $L_{\zeta}$. For $t \in[r]$, and $i, j$ in $[k]$ we define the event $I_{i, j}^{t}$ as follows:

$$
\left.\left.I_{i, j}^{t}=\{(\mathbf{S}, f) \in \Omega,] i, j\right] \subseteq S_{t}\right\} .
$$

Observe that $I_{i, i}^{t}:=\Omega$ because $\left.] i, i\right]=\emptyset$. We also define the event $J_{i, j}^{t}$ by $J_{i, j}^{t}=I_{i, j}^{t}$ if $i \neq j$ and $J_{i, i}^{t}:=\left\{(\mathbf{S}, f), S_{t}=[k]\right\}$. For $i \in[k-1]$ and $j \in[k]$ we define the following generalized events

$$
\begin{aligned}
L_{\alpha, i, j}^{\prime} & =I_{i, j}^{f(i)}-I_{i, j-1}^{f(i)}, \\
L_{\beta, i, j}^{\prime} & =J_{i, j+1}^{f(i)}-J_{i, j}^{f(i)}, \\
L_{\gamma, i, j}^{\prime} & =J_{i, j+1}^{f(i)}-J_{i, j}^{f(i)}-J_{i-1, j+1}^{f(i)}+J_{i-1, j}^{f(i)} .
\end{aligned}
$$

Here and in the following, we consider the subscripts of the events $I_{i, j}^{t}$ and $J_{i, j}^{t}$ cyclically modulo $k$; for instance $J_{0, j}^{t}$ is understood as $J_{k, j}^{t}$.

It is easy to check that for $\zeta$ in $\{\alpha, \beta, \gamma\}$ and for all $i \in[k-1], j \in[k]$ such that $i \neq j$, one has the equivalence of events $L_{\zeta, i, j}^{\prime} \sim L_{\zeta, i, j}$. For instance, $L_{\alpha, i, j}=-E_{\alpha, i, j}$ where

$$
E_{\alpha, i, j}=\left\{i+1, . ., j-1 \in S_{f(i)} \text {, and } j \notin S_{f(i)}\right\}=I_{i, j-1} \backslash I_{i, j}
$$

hence $L_{\alpha, i, j}=-I_{i, j-1} \backslash I_{i, j} \sim I_{i, j}-I_{i, j-1}$. Moreover, for all $i \in[k-1]$,

$$
L_{\zeta, i, i}^{\prime}=-\sum_{j \in[k-1] \backslash\{i\}} L_{\zeta, i, j}^{\prime} \sim-\sum_{j \in[k-1] \backslash\{i\}} L_{\zeta, i, j}=L_{\zeta, i, i} .
$$

Thus, by Lemma 8 the matrices $L_{\zeta}^{\prime}$ and $L_{\zeta}$ have the same $\mathbb{P}$-determinant. Our findings so far are summarized in the following lemma.

Lemma 9. For all $\zeta \in\{\alpha, \beta, \gamma\}$, the probability $P_{\zeta}(\boldsymbol{p}, r)$ defined in Theorem 1 is

$$
P_{\zeta}(\boldsymbol{p}, r)=\frac{\left|\mathcal{S}_{\boldsymbol{p}, r}\right|}{\left|\mathcal{R}_{\boldsymbol{p}, r}\right|} \mathbb{P d e t}\left(L_{\zeta}^{\prime}\right),
$$

where $L_{\zeta}^{\prime}=\left(L_{\zeta, i, j}^{\prime}\right)_{i, j \in[k-1]}$ is the matrix of generalized events defined by (4). 


\subsection{Simplifications of $\mathbb{P}$-determinants}

We will now simplify the determinantal expressions for the probabilities $P_{\zeta}(\mathbf{p}, r)$.

Proposition 10. For all $\zeta \in\{\alpha, \beta, \gamma\}$, the probability $P_{\zeta}(\boldsymbol{p}, r)$ defined in Theorem 1 is

$$
P_{\zeta}(\boldsymbol{p}, r)=\frac{\left|\mathcal{S}_{p, r}\right|}{\left|\mathcal{R}_{p, r}\right|} \mathbb{P} \operatorname{det}\left(M_{\zeta}\right)
$$

where $M_{\zeta}=\left(M_{\zeta, i, j}\right)_{i, j \in[k]}$ is the matrix of generalized events defined by

$$
\begin{aligned}
& M_{\alpha, i, j}=I_{i, j}^{f(i)} \quad \text { if } i \in[k-1] \text {, and } M_{\alpha, k, j}=\Omega \text {, } \\
& M_{\beta, i, j}=J_{i, j+1}^{f(i)} \quad \text { if } i \in[k-1] \text {, and } M_{\beta, k, j}=\Omega \text {, } \\
& M_{\gamma, i, j}=J_{i, j+1}^{f(i)}-J_{i-1, j+1}^{f(i)} \quad \text { if } i \in[k-1] \text {, and } M_{\gamma, k, j}=\Omega \text {. }
\end{aligned}
$$

Example. Let us illustrate Proposition 10 in the case $\zeta=\alpha$ and $k=3$. In this case,

$$
M_{\alpha}=\left(\begin{array}{ccc}
I_{1,1}^{f(1)} & I_{1,2}^{f(1)} & I_{1,3}^{f(1)} \\
I_{2,1}^{f(2)} & I_{2,2}^{f(2)} & I_{2,3}^{f(2)} \\
\Omega & \Omega & \Omega
\end{array}\right)=\left(\begin{array}{ccc}
\Omega & 2 \in S_{f(1)} & 2,3 \in S_{f(1)} \\
1,3 \in S_{f(2)} & \Omega & 3 \in S_{f(2)} \\
\Omega & \Omega & \Omega
\end{array}\right)
$$

hence by definition of the $\mathbb{P}$-determinant,

$$
\begin{aligned}
\mathbb{P d e t}\left(M_{\alpha}\right)= & \mathbb{P}(\Omega)-\mathbb{P}\left(3 \in S_{f(2)}\right)+\mathbb{P}\left(2 \in S_{f(1)} \cap 3 \in S_{f(2)}\right)-\mathbb{P}\left(2,3 \in S_{f(1)}\right) \\
& -\mathbb{P}\left(2 \in S_{f(1)} \cap 1,3 \in S_{f(2)}\right)+\mathbb{P}\left(2,3 \in S_{f(1)} \cap 1,3 \in S_{f(2)}\right) .
\end{aligned}
$$

Proposition 10 asserts that the probability $P_{\alpha}(\mathbf{p}, r)$ that the graph $G_{\alpha}(\mathbf{S}, f)$ is a tree is equal to $\frac{\left|\mathcal{S}_{\mathbf{p}, r}\right|}{\left|\mathcal{R}_{\mathbf{p}, r}\right|} \mathbb{P} \operatorname{det}\left(M_{\alpha}\right)$. We leave as an exercise to prove that the right-hand side of $(5)$ is equal to $\frac{\left|\mathcal{R}_{\mathbf{p}, r}\right|}{\left|\mathcal{S}_{\mathbf{p}, r}\right|} \times\left(1-p_{3} / r\right)$ as predicted by Theorem 1 .

The rest of this section is devoted to the proof of Proposition 10. We first treat in detail the case $\zeta=\alpha$. Given Lemma 9 we only need to prove $\mathbb{P} \operatorname{det}\left(L_{\alpha}^{\prime}\right)=\operatorname{Pdet}\left(M_{\alpha}\right)$, where $L_{\alpha}^{\prime}=\left(I_{i, j}^{f(i)}-I_{i, j-1}^{f(i)}\right)_{i, j \in[k-1]}$. Since $\mathbb{P}$-determinants are alternating in the columns of matrices, we can replace the $j$ th column of $L_{\alpha}^{\prime}$ by the sum of its $j$ first columns without changing the $\mathbb{P}$-determinant. This gives,

$$
\mathbb{P} \operatorname{det}\left(L_{\alpha}^{\prime}\right)=\mathbb{P} \operatorname{det}\left(I_{i, j}^{f(i)}-I_{i, k}^{f(i)}\right)_{i, j \in[k-1]} .
$$

Next, by linearity of the $\mathbb{P}$-determinant in the rows of the matrix, one gets

$$
\mathbb{P} \operatorname{det}\left(L_{\alpha}^{\prime}\right)=\sum_{D \subseteq[k-1]}(-1)^{|D|} \mathbb{P} \operatorname{det}\left(M^{D}\right)
$$

where $M^{D}=\left(M_{i, j}^{D}\right)_{i, j \in[k-1]}$ with $M_{i, j}^{D}=I_{i, k}^{f(i)}$ if $i \in D$ and $M_{i, j}^{D}=I_{i, j}^{f(i)}$ otherwise. We now show that only $k$ of the subsets $D$ contribute to the above sum. 
Lemma 11. If $D \subseteq[k-1]$ contains more than one element, then $\mathbb{P} \operatorname{det}\left(M^{D}\right)=0$.

Proof. We assume that $D$ contains two distinct integers $a$ and $b$ and want to show that $\mathbb{P} \operatorname{det}\left(M^{D}\right)=0$. We will use the expression (2) of $\mathbb{P}$-determinant. By definition, a triple $(\mathbf{S}, f, \pi) \in \Omega_{k-1}$ is in $\Omega\left(M^{D}\right)$ if and only if for all $\left.\left.i \in D,\right] i, k\right] \subseteq S_{f(i)}$ and for all $i \in[k] \backslash D,] i, \pi(i)] \subseteq S_{f(i)}$. Observe that the above conditions for $i=a$ and $i=b$, namely ]$a, k] \subseteq \mathcal{S}_{f(a)}$ and $\left.] b, k\right] \subseteq \mathcal{S}_{f(b)}$, do not depend on the permutation $\pi$. More generally, none of the above conditions is affected by changing the permutation $\pi$ by $\pi \circ(a, b)$, where $(a, b)$ is the transposition of the integers $a$ and $b$. Thus a triple $(\mathbf{S}, f, \pi)$ is in $\Omega\left(M^{D}\right)$ if and only if $\phi(\mathbf{S}, f, \pi):=(\mathbf{S}, f, \pi \circ(a, b))$ is in $\Omega\left(M^{D}\right)$. Thus the mapping $\phi$ is an involution of $\Omega\left(M^{D}\right)$. Moreover, since the involution $\phi$ changes the sign of the permutation $\pi$, we get

$$
\mathbb{P} \operatorname{det}\left(M^{D}\right)=\frac{1}{|\Omega|} \sum_{(\mathbf{S}, f, \pi) \in \Omega\left(M^{D}\right)} \epsilon(\pi)=0,
$$

as claimed.

So far we have shown that

$$
\mathbb{P} \operatorname{det}\left(L_{\alpha}^{\prime}\right)=\mathbb{P} \operatorname{det}\left(M^{\emptyset}\right)-\sum_{a \in[k-1]} \mathbb{P} \operatorname{det}\left(M^{\{a\}}\right) .
$$

Next, we observe that the set of triples $(\mathbf{S}, f, \pi)$ in $\Omega\left(M^{\emptyset}\right)$ can be identified with the set of triples $\left(\mathbf{S}, f, \pi^{\prime}\right)$ in $\Omega\left(M_{\alpha}\right)$ such that $\pi^{\prime}(k)=k$. Indeed, the correspondence is simply obtained by replacing the permutation $\pi$ of $[k-1]$ by the permutation $\pi^{\prime}$ of $[k]$ such that $\pi^{\prime}(k)=k$, and $\pi^{\prime}(i)=\pi(i)$ for all $i$ in $[k-1]$. Similarly, for all $a \in[k-1]$, there is a bijection between the set of triples $(\mathbf{S}, f, \pi)$ in $\Omega\left(M^{\{a\}}\right)$ and the set of triples $\left(\mathbf{S}, f, \pi^{\prime}\right)$ in $\Omega\left(M_{\alpha}\right)$ such that $\pi^{\prime}(a)=k$. Indeed, the bijection is simply obtained by replacing the permutation $\pi$ of $[k-1]$ by the permutation $\pi^{\prime}$ of $[k]$ such that $\pi^{\prime}(a)=k, \pi^{\prime}(k)=\pi(a)$ and $\pi^{\prime}(i)=\pi(i)$ for all $i \neq a$ in $[k-1]$. Observe that this bijection changes the sign of the permutation, hence

$$
\begin{aligned}
\operatorname{Pdet}\left(L_{\alpha}^{\prime}\right) & =\frac{1}{|\Omega|} \sum_{(\mathbf{S}, f, \pi) \in \Omega\left(M^{\emptyset}\right)} \epsilon(\pi)-\sum_{a \in[k-1]} \sum_{(\mathbf{S}, f, \pi) \in \Omega\left(M^{\{a\}}\right)} \epsilon(\pi) \\
& =\frac{1}{|\Omega|} \sum_{\left(\mathbf{S}, f, \pi^{\prime}\right) \in \Omega\left(M_{\alpha}\right)} \epsilon(\pi)=\mathbb{P} \operatorname{det}\left(M_{\alpha}\right) .
\end{aligned}
$$

This completes the proof of Proposition 10 in the case $\zeta=\alpha$.

The proof of Proposition 10 in the case $\zeta=\beta$ (resp. $\zeta=\gamma$ ), is exactly the same as the proof given above for $\zeta=\alpha$, except that the events $I_{i, j}^{t}$ are replaced by $J_{i, j+1}^{t}$ (resp. $\left.J_{i, j+1}^{t}-J_{i-1, j+1}^{t}\right)$.

\section{Computing $\mathbb{P}$-determinants using sign reversing involutions}

In this section we complete the proof of Theorem 1 by computing the $\mathbb{P}$-determinant of the matrices $M_{\zeta}$ for $\zeta \in\{\alpha, \beta, \gamma\}$. 


$$
\left(\begin{array}{cccccccc}
I_{1,1}^{f(1)}-J_{1,1}^{f(1)} & 0 & \cdots & \ldots & \ldots & \cdots & \cdots & 0 \\
0 & I_{2,2}^{f(2)}-J_{2,2}^{f(2)} & 0 & \cdots & \cdots & \cdots & \cdots & 0 \\
\vdots & & \ddots & & & & & \vdots \\
0 & \ldots & 0 & I_{a-2, a-2}^{f(a-2)} J_{a-2, a-2}^{f(a-2)} & 0 & \ldots & \cdots & 0 \\
I_{a-1,1}^{f(a-1)} & I_{a-1,2}^{f(a-1)} & \cdots & I_{a-1, a-2}^{f(a-1)} & \mathbf{E} & I_{a-1, a}^{f(a-1)} & \cdots & I_{a-1, k}^{f(a-1)} \\
I_{a, 1}^{f(a)} & I_{a, 2}^{f(a)} & \cdots & I_{a, a-2}^{f(a)} & I_{a, a-1}^{f(a)} & I_{a, a}^{f(a)} & \cdots & I_{a, k}^{f(a)} \\
\vdots & \vdots & & \vdots & \vdots & \vdots & & \vdots \\
I_{k-1,1}^{f(k-1)} & I_{k-1,2}^{f(k-1)} & \cdots & I_{k-1, a-2}^{f(k-1)} & I_{k-1, a-1}^{f(k-1)} & I_{k-1, a}^{f(k-1)} & \cdots & I_{k-1, k}^{f(k-1)} \\
\Omega & \Omega & \cdots & \Omega & \Omega & \Omega & \cdots & \Omega
\end{array}\right)
$$

Figure 3: The matrix $M^{(a)}$ (resp. $N^{(a)}$ ) is the matrix represented above with the entry $\mathbf{E}$ in position $(a-1, a-1)$ equal to $\mathbf{E}=I_{a-1, a-1}^{f(a-1)}\left(\right.$ resp. $\left.\mathbf{E}=J_{a-1, a-1}^{f(a-1)}\right)$.

\subsection{Computing the $\mathbb{P}$-determinant of the matrix $M_{\alpha}$.}

In this section we compute the $\mathbb{P}$-determinant of the matrix $M_{\alpha}$.

Proposition 12. The $\mathbb{P}$-determinant of the matrix $M_{\alpha}$ defined in Proposition 10 is

$$
\operatorname{Pdet}\left(M_{\alpha}\right)=\frac{\left|\mathcal{R}_{p, r}\right|}{\left|\mathcal{S}_{p, r}\right|} \times\left(1-p_{k} / r\right)
$$

Note that Proposition 12 together with Proposition 10 prove the case $(a)$ of Theorem 1. In order to prove Proposition 12, we first use the multilinearity of $\mathbb{P}$-determinants with respect to rows. For $a \in[k]$, we denote by $M^{(a)}=\left(M_{i, j}^{(a)}\right)_{i, j \in[k]}$ the matrix of generalized events represented in Figure 3, that is, $M_{i, j}^{(a)}=M_{\alpha, i, j}$ if $i \geqslant a-1, M_{i, j}^{(a)}=0$ if $i<a-1$ and $j \neq i$, and $M_{i, i}^{(a)}=I_{i, i}^{f(i)}-J_{i, i}^{f(i)}$ if $i<a-1$. Note that $M^{(1)}=M^{(2)}=M_{\alpha}$. For $a \in\{2,3, \ldots, k-1\}$, we also denote $N^{(a)}=\left(N_{i, j}^{(a)}\right)_{i, j \in[k]}$ the matrix of events which is the same as $M^{(a)}$ except the entry in position $(a-1, a-1)$ is $J_{a-1, a-1}^{f(a-1)}$ (see Figure 3 ).

By linearity of the $\mathbb{P}$-determinant with respect to matrix rows one gets

$$
\mathbb{P} \operatorname{det}\left(M^{(a)}\right)=\mathbb{P} \operatorname{det}\left(N^{(a)}\right)+\mathbb{P} \operatorname{det}\left(M^{(a+1)}\right),
$$

for all $a \in\{2,3, \ldots, k-1\}$. Hence

$$
\mathbb{P} \operatorname{det}\left(M_{\alpha}\right)=\mathbb{P} \operatorname{det}\left(M^{(2)}\right)=\mathbb{P} \operatorname{det}\left(M^{(k)}\right)+\sum_{a=2}^{k-1} \mathbb{P} \operatorname{det}\left(N^{(a)}\right) .
$$

We will now show that $\mathbb{P} \operatorname{det}\left(M^{(k)}\right)=\frac{\left|\mathcal{R}_{\mathbf{p}, r}\right|}{\left|\mathcal{S}_{\mathbf{p}, r}\right|} \times\left(1-p_{k} / r\right)$, and $\mathbb{P} \operatorname{det}\left(N^{(a)}\right)=0$ for all $a \in\{2,3, \ldots, k-1\}$, thereby proving Proposition 12 . 
Lemma 13. The $\mathbb{P}$-determinant of the matrix $M^{(k)}$ is $\mathbb{P} \operatorname{det}\left(M^{(k)}\right)=\frac{\left|\mathcal{R}_{\boldsymbol{p}, r}\right|}{\left|\mathcal{S}_{\boldsymbol{p}, r}\right|} \times\left(1-p_{k} / r\right)$.

Proof. Since all the entries in the last row of $M^{(k)}$ are equal to $\Omega$, one gets

$$
\mathbb{P} \operatorname{det}\left(M^{(k)}\right)=\sum_{\pi \in \mathfrak{S}_{k}} \epsilon(\pi) \mathbb{P}\left(\bigcap_{i=1}^{k-1} M_{i, \pi(i)}^{(k)}\right) .
$$

Moreover, the only permutations $\pi$ contributing to the sum are the identity $\pi=\operatorname{Id}_{k}$ and the transposition $\pi=(k-1, k)$, which gives

$$
\begin{aligned}
\mathbb{P} \operatorname{det}\left(M^{(k)}\right) & =\mathbb{P}\left(\bigcap_{i=1}^{k-2}\left(I_{i, i}^{f(i)}-J_{i, i}^{f(i)}\right) \cap I_{k-1, k-1}^{f(k-1)}\right)-\mathbb{P}\left(\bigcap_{i=1}^{k-2}\left(I_{i, i}^{f(i)}-J_{i, i}^{f(i)}\right) \cap I_{k-1, k}^{f(k-1)}\right) \\
& =\mathbb{P}\left(\bigcap_{i=1}^{k-2}\left(I_{i, i}^{f(i)}-J_{i, i}^{f(i)}\right) \cap\left(I_{k-1, k-1}^{f(k-1)}-I_{k-1, k}^{f(k-1)}\right)\right) .
\end{aligned}
$$

By definition, $I_{i, i}^{f(i)}=\Omega$, and $J_{i, i}^{f(i)}$ is the event " $S_{f(i)}=[k]$ ". Hence the generalized event $\left(I_{i, i}^{f(i)}-J_{i, i}^{f(i)}\right)$ is equivalent to the event " $S_{f(i)} \neq[k]$ ". Moreover, $I_{k-1, k}^{f(k-1)}$ is the event " $k \in S_{f(k-1)}$ ". Hence the generalized event $\left(I_{k-1, k-1}^{f(k-1)}-I_{k-1, k}^{f(k-1)}\right)$ is equivalent to the event " $k \notin S_{f(k-1)}$ ". Thus $\mathbb{P} \operatorname{det}\left(M^{(k)}\right)$ is equal to the probability of the event "for all $i \in[k-1]$, the subset $S_{f(i)}$ is a proper subset of $[k]$ and $k \notin S_{f(k-1)}$ ". And since $f$ is a surjection from $[k-1]$ to $[r]$, this event is "the subsets $S_{1}, \ldots, S_{r}$ are proper subsets of $[k]$ and $k \notin S_{f(k-1)} "$. Thus

$$
\mathbb{P} \operatorname{det}\left(M^{(k)}\right)=\mathbb{P}\left(\mathbf{S} \in \mathcal{R}_{\mathbf{p}, r}\right) \times \mathbb{P}\left(k \notin S_{f(k-1)} \mid \mathbf{S} \in \mathcal{R}_{\mathbf{p}, r}\right) .
$$

Lastly, the conditional probability $\mathbb{P}\left(k \notin S_{f(k-1)} \mid \mathbf{S} \in \mathcal{R}_{\mathbf{p}, r}\right)$ is equal to $1-p_{k} / r$ since for any tuple $\mathbf{S}=\left(S_{1} \ldots, S_{r}\right) \in \mathcal{R}_{\mathbf{p}, r}$ there are exactly $p_{k}$ of the $r$ subsets $S_{1}, \ldots, S_{r}$ containing the integer $k$.

It remains to prove that $\mathbb{P} \operatorname{det}\left(N^{(a)}\right)=0$ for all $a \in\{2,3, \ldots, k-1\}$. For $D \subseteq[a-2]$, we denote by $N^{a, D}=\left(N_{i, j}^{a, D}\right)_{i, j \in[k]}$ the matrix of generalized events defined by $N_{i, j}^{a, D}=N_{i, j}^{(a)}$ if $i \geqslant a-1$ or $j \neq i, N_{i, i}^{a, D}=J_{i, i}^{f(i)}$ if $i \in D$, and $N_{i, i}^{a, D}=I_{i, i}^{f(i)}$ if $i \in[a-2] \backslash D$. By multilinearity of the $\mathbb{P}$-determinant in the rows of the matrix, one gets

$$
\mathbb{P} \operatorname{det}\left(N^{(a)}\right)=\sum_{D \subseteq[a-2]}(-1)^{|D|} \mathbb{P} \operatorname{det}\left(N^{a, D}\right) .
$$

It now suffices to prove the following lemma:

Lemma 14. For all $a \in\{2,3, \ldots, k-1\}$, and for all $D \subseteq[a-2], \mathbb{P} \operatorname{det}\left(N^{a, D}\right)=0$. 
Intuition for the upcoming proof of Lemma 14. We aim at proving $\mathbb{P} \operatorname{det}\left(N^{a, D}\right)=0$ using (2). For this, it suffices to find an involution on the set of triples $(\mathbf{S}, f, \pi)$ in $\Omega\left(N^{a, D}\right)$ changing the sign of the permutation $\pi$. Now, the hope for finding such an involution is based on the observation that the events appearing in the $(a-1)$ th and ath rows of the matrix $N^{a, D}$ are very similar: for all $j$ in $[k]$ the events $N_{a-1, j}^{a, D}$ and $N_{a, j}^{a, D}$ are respectively " $\{a\} \cup] a, j] \subseteq S_{f(a-1)}$ " and " $\left.] a, j\right] \subseteq S_{f(a)}$ ". This implies that changing the permutation $\pi$ into $\pi^{\prime}=\pi \circ(a-1, a)$ and the surjection $f$ into $f^{\prime}=f \circ(a-1, a)$ (where $(a-1, a)$ is the transposition of the integers $a-1$ and $a$ ) will only change the requirement " $a \in S_{f(a-1)}$ " into " $a \in S_{f(a)}$ ". Now, this can be achieved by also transferring the element $a$ from $S_{f(a-1)}$ to $S_{f(a)}$; and this is what is done in the proof of Lemma 17 below. However, this trick does not work for all triples $(\mathbf{S}, f, \pi) \in \Omega\left(N^{a, D}\right)$ because the requirement " $a \in S_{f(a-1)}$ " might still hold for $\left(\mathbf{S}, f^{\prime}, \pi^{\prime}\right)$. Indeed, this happens if there is $b \in[k-1]$ such that $f(b)=f(a-1)$ and $a \in] b, \pi(b)]$. In those cases, one would think about changing $\pi$ into $\pi \circ(a-1, b)$ instead; and this is the intuition behind the proof of Lemmas 18 and 19 below. However, this does not work in every case, and one has to deal with a few pathological cases on the side. We mention lastly that there exist matrices of generalized events having a non-zero $\mathbb{P}$-determinant but whose $(a-1)$ th and ath rows coincide with those of $N^{a, D}$.

In order to prove Lemma 14 we need some notation. We fix an integer $a \in\{2,3, \ldots, k-$ $1\}$ and a subset $D \subseteq[a-2]$ We define $\mathfrak{S}_{k, a}$ as the set of permutations $\pi$ of $[k]$ such that $\pi(i)=i$ for all $i \in[a-2]$. We also define some subsets $K_{i, j}$ of $[k]$ by setting $K_{i, i}=\emptyset$ if $i \in[a-2] \backslash D, K_{i, i}=[k]$ if $i \in D \cup\{a-1\}$, and $\left.\left.K_{i, j}=\right] i, j\right]$ if $i \in\{a-1, \ldots, k-1\}, j \in[k]$ and $(i, j) \neq(a-1, a-1)$. By definition, a triple $(\mathbf{S}, f, \pi) \in \Omega_{k}$ is in $\Omega\left(N^{a, D}\right)$ if and only if the permutation $\pi$ is in $\mathfrak{S}_{k, a}$, and for all $i \in[k-1]$ the subset $K_{i, \pi(i)}$ is contained in $S_{f(i)}$. For $\pi \in \mathfrak{S}_{k, a}, f$ a surjection from $[k-1]$ to $[r]$, and $t \in[r]$ we define the subset $R_{f, \pi, t}$ of $[k]$ by

$$
R_{f, \pi, t}=\bigcup_{i \in[k-1], f(i)=t} K_{i, \pi(i)} .
$$

Observe that a triple $(\mathbf{S}, f, \pi) \in \Omega_{k}$ is in $\Omega\left(N^{a, D}\right)$ if and only if the permutation $\pi$ is in $\mathfrak{S}_{k, a}$ and for all $t \in[r]$ the subset $R_{f, \pi, t}$ is contained in $S_{t}$. Lastly for $j \in[k-1]$ we denote

$$
H_{f, \pi, j}=\bigcup_{i \in[k-1] \backslash\{j\}, f(i)=f(j)} K_{i, \pi(i)}
$$

so that $R_{f, \pi, f(j)}=H_{f, \pi, j} \cup K_{j, \pi(j)}$.

We now define a partition of the set $\Omega\left(N^{a, D}\right)$, by declaring that a triple $(\mathbf{S}, f, \pi) \in$ $\Omega\left(N^{a, D}\right)$ is in

- $\mathcal{W}$ if $f(a-1)=f(a)$,

- $\mathcal{X}$ if $f(a-1) \neq f(a), a \notin H_{f, \pi, a-1}$, and $a \notin H_{f, \pi, a}$,

- $\mathcal{Y}$ if $f(a-1) \neq f(a), a \notin H_{f, \pi, a-1}$, and $a \in H_{f, \pi, a}$,

- $\mathcal{Z}$ if $f(a-1) \neq f(a), a \in H_{f, \pi, a-1}$. 
Since $\Omega\left(N^{a, D}\right)=\mathcal{W} \uplus \mathcal{X} \uplus \mathcal{Y} \uplus \mathcal{Z}$, Equation (2) gives

$$
\mathbb{P} \operatorname{det}\left(N^{a, D}\right)=\frac{W+X+Y+Z}{|\Omega|},
$$

where

$$
W=\sum_{(\mathbf{S}, f, \pi) \in \mathcal{W}} \epsilon(\pi), \quad X=\sum_{(\mathbf{S}, f, \pi) \in \mathcal{X}} \epsilon(\pi), \quad Y=\sum_{(\mathbf{S}, f, \pi) \in \mathcal{Y}} \epsilon(\pi), \quad \text { and } \quad Z=\sum_{(\mathbf{S}, f, \pi) \in \mathcal{Z}} \epsilon(\pi) .
$$

We will now show that $W=X=Y=Z=0$.

Remark 15. In the particular case $r=k-1$, the surjection $f$ is a bijection and $H_{f, \pi, j}=\emptyset$ for all $j$. Therefore, in this case $\mathcal{X}=\Omega\left(N^{a, D}\right)$, while $\mathcal{W}=\mathcal{Y}=\mathcal{Z}=\emptyset$.

Lemma 16. The sum $W$ defined in (8) is equal to 0.

Proof. We consider the mapping $\phi$ defined on $\mathcal{W}$ by setting $\phi(\mathbf{S}, f, \pi)=\left(\mathbf{S}, f, \pi^{\prime}\right)$, where $\pi^{\prime}=\pi \circ(a-1, a)$ (where $(a-1, a)$ is the transposition of the integers $a-1$ and $a$ ). Since $\phi$ changes the sign of the permutation $\pi$, it suffices to prove that $\phi$ is an involution on $\mathcal{W}$. Clearly this amounts to proving that $\left(\mathbf{S}, f, \pi^{\prime}\right) \in \mathcal{W}$. It is clear that $R_{f, \pi^{\prime}, t}=R_{f, \pi, t}$ for all $t \in[r] \backslash\{f(a)\}$. Moreover, remembering $\left.\left.K_{a-1, j}=\{a\} \cup\right] a, j\right]$ and $\left.\left.K_{a, j}=\right] a, j\right]$ for all $j \in[k]$ gives

$$
\left.\left.\left.\left.K_{a-1, \pi(a-1)} \cup K_{a, \pi(a)}=\{a\} \cup\right] a, \pi(a-1)\right] \cup\right] a, \pi(a)\right]=K_{a-1, \pi^{\prime}(a-1)} \cup K_{a, \pi^{\prime}(a)},
$$

hence $R_{f, \pi^{\prime}, f(a)}=R_{f, \pi, f(a)}$. Thus, for all $t \in[r], R_{f, \pi^{\prime}, t}=R_{f, \pi, t} \subseteq S_{t}$, that is, $\left(\mathbf{S}, f, \pi^{\prime}\right) \in$ $\Omega\left(N^{a, D}\right)$. Since $f(a-1)=f(a)$ we have $\left(\mathbf{S}, f, \pi^{\prime}\right) \in \mathcal{W}$ as wanted.

Lemma 17. The sum $X$ defined in (8) is equal to 0.

Proof. We first define a mapping $\phi$ on $\mathcal{X}$. Let $(\mathbf{S}, f, \pi)$ be in $\mathcal{X}$. Note that $a \in S_{f(a-1)}$ (because $\left.a \in K_{a-1, \pi(a-1)} \subseteq S_{f(a-1)}\right)$. We now denote by $\mathbf{S}^{\prime}=\left(S_{1}^{\prime}, \ldots, S_{r}^{\prime}\right)$ the tuple obtained from $\mathbf{S}=\left(S_{1}, \ldots, S_{r}\right)$ by exchanging the presence or absence of the integer $a$ between the subsets $S_{f(a-1)}$ and $S_{f(a)}$. More precisely, $\mathbf{S}^{\prime}=\mathbf{S}$ if $a \in S_{f(a)}$, and otherwise $S_{f(a)}^{\prime}=S_{f(a)} \cup\{a\}, S_{f(a-1)}^{\prime}=S_{f(a-1)} \backslash\{a\}$, and $S_{i}^{\prime}=S_{i}$ for all $i \notin\{f(a-1), f(a)\}$. We now define a mapping $\phi$ on $\mathcal{X}$ by setting $\phi(\mathbf{S}, f, \pi)=\left(\mathbf{S}^{\prime}, f^{\prime}, \pi^{\prime}\right)$, where $\mathbf{S}^{\prime}$ is defined as above, $f^{\prime}=f \circ(a-1, a)$, and $\pi^{\prime}=\pi \circ(a-1, a)$. We observe the following identities:

$$
\begin{array}{r}
K_{a-1, \pi^{\prime}(a-1)}=K_{a, \pi(a)} \cup\{a\}, \quad K_{a, \pi^{\prime}(a)}=K_{a-1, \pi(a-1)} \backslash\{a\}, \\
H_{f^{\prime}, \pi^{\prime}, a-1}=H_{f, \pi, a}, \text { and } H_{f^{\prime}, \pi^{\prime}, a}=H_{f, \pi, a-1} .
\end{array}
$$

We will now prove that $\phi$ is an involution on $\mathcal{X}$. Observe that this immediately implies that $X=0$ because $\phi$ changes the sign of the permutation $\pi$.

Claim. The mapping $\phi$ is an involution on $\mathcal{X}$.

Proof of the claim. Clearly, it suffices to prove that if $(\boldsymbol{S}, f, \pi)$ is in $\mathcal{X}$ then $\left(\boldsymbol{S}^{\prime}, f^{\prime}, \pi^{\prime}\right)=$ $\phi(\boldsymbol{S}, f, \pi)$ is in $\mathcal{X}$. Let $(\mathbf{S}, f, \pi) \in \mathcal{X}$. Clearly, $\mathbf{S}^{\prime}$ is in $\mathcal{S}_{\mathbf{p}, r}, f^{\prime}$ is a surjection from $[k-1]$ 
to $[r]$, and $\pi^{\prime}$ is in $\mathfrak{S}_{k, a}$. Hence $\phi(\mathbf{S}, f, \pi)$ is in $\Omega_{k}$. Next, we show that $\left(\mathbf{S}^{\prime}, f^{\prime}, \pi^{\prime}\right)$ is in $\Omega\left(N^{a, D}\right)$, that is, $R_{f^{\prime}, \pi^{\prime}, t} \subseteq S_{t}^{\prime}$ for all $t \in[r]$. First, for $t \notin\{f(a-1), f(a)\}$, we have $R_{f^{\prime}, \pi^{\prime}, t}=R_{f, \pi, t}$ and $S_{t}^{\prime}=S_{t}$, hence $R_{f^{\prime}, \pi^{\prime}, t} \subseteq S_{t}^{\prime}$. For $t=f(a-1)=f^{\prime}(a)$, Equations (9) and (10) give

$$
R_{f^{\prime}, \pi^{\prime}, t}=H_{f^{\prime}, \pi^{\prime}, a} \cup K_{a, \pi^{\prime}(a)}=H_{f, \pi, a-1} \cup K_{a-1, \pi(a-1)} \backslash\{a\}=R_{f, \pi, t} \backslash\{a\} .
$$

Moreover, the set $S_{t}^{\prime}$ does not differ from $S_{t}$ except maybe for the presence or absence of $a$, hence $R_{f^{\prime}, \pi^{\prime}, t} \subseteq S_{t}^{\prime}$. Lastly, for $t=f(a)=f^{\prime}(a-1)$, Equations (9) and (10) give

$$
R_{f^{\prime}, \pi^{\prime}, t}=H_{f^{\prime}, \pi^{\prime}, a-1} \cup K_{a-1, \pi^{\prime}(a-1)}=H_{f, \pi, a} \cup K_{a, \pi(a)} \cup\{a\}=R_{f, \pi, t} \cup\{a\} .
$$

Moreover, $S_{t}^{\prime}=S_{t} \cup\{a\}$, hence $R_{f^{\prime}, \pi^{\prime}, t} \subseteq S_{t}^{\prime}$. This shows that $\left(\mathbf{S}^{\prime}, f^{\prime}, \pi^{\prime}\right)$ is in $\Omega\left(N^{a, D}\right)$. Furthermore, $\left(\mathbf{S}^{\prime}, f^{\prime}, \pi^{\prime}\right)$ is in $\mathcal{X}$ because of (10) (and the fact that $(\mathbf{S}, f, \pi)$ in $\mathcal{X}$ ). This completes the proof of the claim, hence $X=0$.

Lemma 18. The sum $Y$ defined in (8) is equal to 0.

Proof. We define a partition of $\mathcal{Y}$ by declaring that a triple $(\mathbf{S}, f, \pi) \in \mathcal{Y}$ is in

- $\mathcal{Y}_{1}$ if $H_{f, \pi, a} \cup K_{a, \pi(a)}=[k]$, and $H_{f, \pi, a} \cup K_{a, \pi(k)}=[k]$,

- $\mathcal{Y}_{2}$ if $H_{f, \pi, a} \cup K_{a, \pi(a)}=[k]$, and $H_{f, \pi, a} \cup K_{a, \pi(k)} \neq[k]$

- $\mathcal{Y}_{3}$ if $H_{f, \pi, a} \cup K_{a, \pi(a)} \neq[k]$.

Next, we define a mapping $\phi$ on $\mathcal{Y}$ by setting $\phi(\mathbf{S}, f, \pi)=\left(\mathbf{S}, f, \pi^{\prime}\right)$, with

- $\pi^{\prime}=\pi \circ(a, k)$ if $(\mathbf{S}, f, \pi) \in \mathcal{Y}_{1}$,

- $\pi^{\prime}=\pi \circ(b, k)$ if $(\mathbf{S}, f, \pi) \in \mathcal{Y}_{2}$,

- $\pi^{\prime}=\pi \circ(a, b)$ if $(\mathbf{S}, f, \pi) \in \mathcal{Y}_{3}$,

where $b$ is the greatest integer in $[k-1] \backslash\{a\}$ such that $f(b)=f(a)$ and $a \in K_{b, \pi(b)}$ (such an integer $b$ necessarily exists since $a \in H_{f, \pi, a}$ ). Since the mapping $\phi$ clearly changes the sign of the permutation $\pi$, showing that $\phi$ is an involution on $\mathcal{Y}$ would imply $Y=0$. In the rest of this proof we show that $\phi$ is an involution on $\mathcal{Y}$ and in fact an involution on each of the subsets $\mathcal{Y}_{1}, \mathcal{Y}_{2}, \mathcal{Y}_{3}$.

Claim 1. The mapping $\phi$ described above is an involution on $\mathcal{Y}_{1}$.

Proof of Claim 1. It suffices to prove that if $(\mathbf{S}, f, \pi)$ is in $\mathcal{Y}_{1}$ then $\left(\mathbf{S}, f, \pi^{\prime}\right)=\phi(\mathbf{S}, f, \pi)$ is in $\mathcal{Y}_{1}$. First observe that by definition of $\mathcal{Y}_{1}$,

$$
R_{f, \pi, f(a)}=[k]=R_{f, \pi^{\prime}, f(a)} .
$$

Hence $R_{f, \pi, t}=R_{f, \pi^{\prime}, t}$ for all $t \in[r]$. Thus $R_{f, \pi^{\prime}, t}=R_{f, \pi, t} \subseteq S_{t}$, that is, $\left(\mathbf{S}, f, \pi^{\prime}\right)$ is in $\Omega\left(N^{a, D}\right)$. Next, we show that $\left(\mathbf{S}, f, \pi^{\prime}\right) \in \mathcal{Y}$. Since $(\mathbf{S}, f, \pi)$ is in $\mathcal{Y}$ we get $a \notin$ 
$H_{f, \pi, a}=H_{f, \pi^{\prime}, a-1}$. Moreover, $a \in H_{f, \pi^{\prime}, a}$ because $a \in[k]=R_{f, \pi^{\prime}, f(a)}$ but $a \notin K_{a, \pi^{\prime}(a)}$. Thus $\left(\mathbf{S}, f, \pi^{\prime}\right) \in \mathcal{Y}$. Lastly, $(\mathbf{S}, f, \pi) \in \mathcal{Y}_{1}$ clearly implies $\left(\mathbf{S}, f, \pi^{\prime}\right) \in \mathcal{Y}_{1}$. This completes the proof of Claim 1 .

Claim 2. The mapping $\phi$ is an involution on $\mathcal{Y}_{2}$.

Proof of Claim 2. Let $(\mathbf{S}, f, \pi)$ be in $\mathcal{Y}_{2}$. We need to prove that $\left(\mathbf{S}, f, \pi^{\prime}\right)=\phi(\mathbf{S}, f, \pi)$ is in $\mathcal{Y}_{2}$ and that $b$ is also the greatest integer in $[k-1] \backslash\{a\}$ such that $f(b)=f(a)$ and $a \in K_{b, \pi^{\prime}(b)}$. First observe that $b>a-2$, otherwise we would have $\pi(b)=b$ and $K_{b, \pi(b)}$ equals $\emptyset$ or $[k]$ but either is impossible (indeed $K_{b, \pi(b)} \neq \emptyset$ because $a \in K_{b, \pi(b)}$, and $K_{b, \pi(b)} \neq[k]$ because $(\mathbf{S}, f, \pi)$ is in $\left.\mathcal{Y}_{2}\right)$, hence in particular $\pi^{\prime}=\pi \circ(b, k)$ is in $\mathfrak{S}_{a, k}$.

We now show that $\left(\mathbf{S}, f, \pi^{\prime}\right)$ is in $\Omega\left(N^{a, D}\right)$, that is, $R_{f, \pi^{\prime}, t} \subseteq S_{t}$ for all $t \in[r]$. First, for $t \neq f(a)$, one has $R_{f, \pi^{\prime}, t}=R_{f, \pi, t} \subseteq S_{t}$. Second, for $t=f(a)$, we have

$$
[k]=H_{f, \pi, a} \cup K_{a, \pi(a)}=R_{f, \pi, f(a)} \subseteq S_{f(a)} .
$$

Hence, $S_{f(a)}=[k]$ and $R_{f, \pi^{\prime}, f(a)} \subseteq S_{f(a)}$. Thus $\left(\mathbf{S}, f, \pi^{\prime}\right)$ is in $\Omega\left(N^{a, D}\right)$.

We now prove that $\left(\mathbf{S}, f, \pi^{\prime}\right)$ is in $\mathcal{Y}_{2}$. Let $c$ be the unique integer in $[k] \backslash H_{f, \pi, a}$ such that $] c, a] \subseteq H_{f, \pi, a}$ (the integer $c$ necessarily exists since $a \in H_{f, \pi, a} \neq[k]$ ). We will show

$$
a \leqslant \pi(k)<c \leqslant b<k, \text { and } a \leqslant \pi(b)<c .
$$

Since $b \notin[a-2]$, and $b \neq\{a-1, a\}$, we get $a<b<k$. Moreover, by definition of $b$ and $c$, we get $] b, a] \subseteq] c, a]$, hence $a<c \leqslant b$. Furthermore, we know $] c, a] \cup] a, \pi(k)] \subseteq$ $\left.\left.H_{f, \pi, a} \cup\right] a, \pi(k)\right] \neq[k]$, hence $a \leqslant \pi(k)<c$. Lastly, $\left.\left.a \in\right] b, \pi(b)\right]$ and $\left.\left.\left.] c, b\right] \cup\right] b, \pi(b)\right] \subseteq$ $H_{f, \pi, a} \neq[k]$, hence $a \leqslant \pi(b)<c$. Thus (11) holds. It implies $\left.\left.a \in\right] b, \pi(k)\right] \subset H_{f, \pi^{\prime}, a}$. Thus $\left(\mathbf{S}, f, \pi^{\prime}\right)$ is in $\mathcal{Y}$. Moreover $\left.\left.c \notin\right] b, \pi(k)\right]$, hence $c \notin H_{f, \pi^{\prime}, a}$. Thus $H_{f, \pi^{\prime}, a} \neq[k]$. We also know $\left.\left.H_{f, \pi, a} \cup\right] a, \pi(a)\right]=[k]$, hence $\left.\left.c \in\right] a, \pi(a)\right]$, which implies $\left.\left.\left.] a, c\right] \subseteq\right] a, \pi(a)\right]$. Moreover,

$$
\begin{gathered}
] c, b] \subseteq] c, a] \subseteq H_{f, \pi, a} \text { and } \pi(b)<c \text {, hence }\right] c, b\right] \subseteq \bigcup_{i \in[k-1] \backslash\{a, b\}, f(i)=f(a)} K_{i, \pi(i)} \text {. Hence, } \\
\left.\left.\left.\left.\left.\left.\left.\left.H_{f, \pi^{\prime}, a} \cup\right] a, \pi(a)\right] \supseteq\right] c, b\right] \cup\right] b, a\right] \cup\right] a, c\right]=[k] .
\end{gathered}
$$

Thus $\left(\mathbf{S}, f, \pi^{\prime}\right)$ is in $\mathcal{Y}_{2}$. Lastly, we know $\left.\left.a \in\right] b, \pi(k)\right]=K_{b, \pi^{\prime}(b)}$, hence $b$ is the greatest integer in $[k-1] \backslash\{a\}$ such that $f(b)=f(a)$ and $a \in K_{b, \pi^{\prime}(b)}$. This shows that $\phi$ is an involution on $\mathcal{Y}_{2}$ and completes the proof of Claim 2.

Claim 3. The mapping $\phi$ is an involution on $\mathcal{Y}_{3}$.

Proof of Claim 3. Let $(\mathbf{S}, f, \pi)$ be in $\mathcal{Y}_{3}$. We need to prove that $\left(\mathbf{S}, f, \pi^{\prime}\right)=\phi(\mathbf{S}, f, \pi)$ is in $\mathcal{Y}_{3}$ and that $b$ is the greatest integer in $[k-1] \backslash\{a\}$ such that $f(b)=f(a)$ and $a \in K_{b, \pi^{\prime}(b)}$. First observe that $b \notin[a-2]$ (indeed $K_{b, \pi(b)} \neq \emptyset$ because $a \in K_{b, \pi(b)}$ and $K_{b, \pi(b)} \neq[k]$ because $(\mathbf{S}, f, \pi)$ is in $\left.\mathcal{Y}_{3}\right)$, hence $\pi^{\prime}=\pi \circ(a, b)$ is in $\mathfrak{S}_{a, k}$. We now show

$$
a \leqslant \pi(a)<b<k, \text { and } a \leqslant \pi(b)<b .
$$

Since $b \notin[a-2]$, and $b \neq\{a-1, a\}$, we get $a<b<k$. Moreover, we know $a \in] b, \pi(b)]$, hence $a \leqslant \pi(b)<b$. Moreover, we know $\left.\left.] a, \pi(a)] \cup] b, \pi(b)] \subseteq H_{f, \pi, a} \cup\right] a, \pi(a)\right] \neq[k]$, hence $a \leqslant \pi(a)<b$. Thus (12) holds. It implies

$$
] a, \pi^{\prime}(a)\right] \cup\right] b, \pi^{\prime}(b)\right]=\right] b, \max (\pi(a), \pi(b))\right]=\right] a, \pi(a)\right] \cup\right] b, \pi(b)\right]
$$


hence $R_{f, \pi^{\prime}, f(a)}=R_{f, \pi, f(a)}$. Thus, for all $t \in[r], R_{f, \pi^{\prime}, t}=R_{f, \pi, t} \subseteq S_{t}$, that is, $\left(\mathbf{S}, f, \pi^{\prime}\right)$ is in $\Omega\left(N^{a, D}\right)$.

Equation (12) also implies $a \in] b, \pi(a)] \subseteq H_{f, \pi^{\prime}, a}$. Thus $\left(\mathbf{S}, f, \pi^{\prime}\right)$ is in $\mathcal{Y}$. Moreover, (13) gives $\left.\left.\left.\left.H_{f, \pi^{\prime}, a} \cup\right] a, \pi^{\prime}(a)\right]=H_{f, \pi, a} \cup\right] a, \pi(a)\right] \neq[k]$. Thus $\left(\mathbf{S}, f, \pi^{\prime}\right)$ is in $\mathcal{Y}_{3}$. Lastly, $a \in] b, \pi(a)]=K_{b, \pi^{\prime}(b)}$, hence $b$ is the greatest integer in $[k-1] \backslash\{a\}$ such that $f(b)=f(a)$ and $a \in K_{b, \pi^{\prime}(b)}$. This shows that $\phi$ is an involution on $\mathcal{Y}_{3}$ and completes the proof of Claim 3.

Claims 1,2,3 imply Lemma 18.

Lemma 19. The sum $Z$ defined in (8) is equal to 0.

Proof. The proof of Lemma 19 is very similar to the proof of Lemma 18 (actually, it is identical except " $a$ " is replaced by " $a-1$ " in certain places). We first define a partition of $\mathcal{Z}$ by declaring that a triple $(\mathbf{S}, f, \pi) \in \mathcal{Z}$ is in

- $\mathcal{Z}_{1}$ if $H_{f, \pi, a-1} \cup K_{a-1, \pi(a-1)}=[k]$, and $H_{f, \pi, a-1} \cup K_{a-1, \pi(k)}=[k]$,

- $\mathcal{Z}_{2}$ if $H_{f, \pi, a-1} \cup K_{a-1, \pi(a-1)}=[k]$, and $H_{f, \pi, a-1} \cup K_{a-1, \pi(k)} \neq[k]$,

- $\mathcal{Z}_{3}$ if $H_{f, \pi, a-1} \cup K_{a-1, \pi(a-1)} \neq[k]$.

Next, we define a mapping $\phi$ on $\mathcal{Z}$ by setting $\phi(\mathbf{S}, f, \pi)=\left(\mathbf{S}, f, \pi^{\prime}\right)$, with

- $\pi^{\prime}=\pi \circ(a-1, k)$ if $(\mathbf{S}, f, \pi) \in \mathcal{Z}_{1}$,

- $\pi^{\prime}=\pi \circ(b, k)$ if $(\mathbf{S}, f, \pi) \in \mathcal{Z}_{2}$,

- $\pi^{\prime}=\pi \circ(a-1, b)$ if $(\mathbf{S}, f, \pi) \in \mathcal{Z}_{3}$,

where $b$ is the greatest integer in $[k-1] \backslash\{a-1\}$ such that $f(b)=f(a-1)$ and $a \in K_{b, \pi(b)}$ (such an integer $b$ necessarily exists since $a \in H_{f, \pi, a-1}$ ). Since the mapping $\phi$ clearly changes the sign of the permutation $\pi$, showing that $\phi$ is an involution on $\mathcal{Z}$ would imply $Z=0$. Actually, the proof that $\phi$ is an involution on each of the subsets $\mathcal{Z}_{1}, \mathcal{Z}_{2}$, and $\mathcal{Z}_{3}$ is almost identical to the one of Lemma 18 (for instance, the identities (11) and (12) still hold with $c$ the integer in $[k] \backslash H_{f, \pi, a-1}$ such that $\left.\left.] c, a\right] \subseteq H_{f, \pi, a-1}\right)$ and is left to the reader.

Lemmas 17, 18, 19 imply Lemma 14. Thus, by (7), $\operatorname{Pdet}\left(N^{(a)}\right)=0$ for all $a \in$ $\{2,3, \ldots, k-1\}$. This together with Equation (6) and Lemma 13 complete the proof of Proposition 12, hence of the case $(a)$ of Theorem 1.

\subsection{Computing the $\mathbb{P}$-determinant of the matrix $M_{\beta}$.}

In this section we compute the $\mathbb{P}$-determinant of the matrix $M_{\beta}$.

Proposition 20. The $\mathbb{P}$-determinant of the matrix $M_{\beta}$ defined in Proposition 10 is

$$
\mathbb{P} \operatorname{det}\left(M_{\beta}\right)=\frac{\left|\mathcal{R}_{q, r-1}\right|}{\left|\mathcal{S}_{p, r}\right|}
$$

where $\boldsymbol{q}=\left(p_{1}, p_{2}-1, p_{3}-1, \ldots, p_{k}-1\right)$. 


$$
\left(\begin{array}{ccccccc}
J_{1,1}^{f(1)} & \cdots & J_{1, a}^{f(1)} & J_{1, a+1}^{f(1)} & \cdots & \cdots & J_{1, k}^{f(1)} \\
\vdots & & \vdots & \vdots & & & \vdots \\
J_{a-1,1}^{f(a-1)} & \cdots & J_{a-1, a}^{f(a-1)} & J_{a-1, a+1}^{f(a-1)} & \cdots & \cdots & J_{a-1, k}^{f(a-1)} \\
J_{a, 1}^{f(a)} & \cdots & \mathbf{E} & J_{a, a+1}^{f(a)} & \cdots & \cdots & J_{a, k}^{f(a)} \\
0 & \cdots & 0 & J_{a+1, a+1}^{f(a+1)}-I_{a+1, a+1}^{f(a+1)} & 0 & \cdots & 0 \\
\vdots & & & & \ddots & & \vdots \\
0 & \cdots & \cdots & \cdots & 0 & J_{k-1, k-1}^{f(k-1)}-I_{k-1, k-1}^{f(k-1)} & 0 \\
\Omega & \cdots & \Omega & \Omega & \cdots & \Omega & \Omega
\end{array}\right)
$$

Figure 4: The matrix $M^{(a)}$ (resp. $N^{(a)}$ ) is the matrix represented above with the entry $\mathbf{E}$ in position $(a, a)$ equal to $\mathbf{E}=J_{a, a}^{f(a)}$ (resp. $\left.\mathbf{E}=I_{a, a}^{f(a)}\right)$.

Note that Proposition 20 together with Proposition 10 prove the case $(b)$ of Theorem 1. We now sketch the proof of Proposition 20 which is very similar to the proof of Proposition 12. We first use some column and row operations on the matrix $M_{\beta}$. Let $M_{\beta}^{\prime}=\left(M_{\beta, i, j}^{\prime}\right)_{i, j \in[k]}$, where $M_{\beta, i, j}^{\prime}=J_{i, j}^{f(i)}$ if $i \neq k$ and $M_{\beta, k, j}^{\prime}=\Omega$. Since the matrix $M_{\beta}^{\prime}$ is obtained from $M_{\beta}$ by reordering its columns cyclically we get

$$
\mathbb{P} \operatorname{det}\left(M_{\beta}\right)=(-1)^{k-1} \mathbb{P} \operatorname{det}\left(M_{\beta}^{\prime}\right) \text {. }
$$

For $a \in[k-1]$, we denote by $M^{(a)}=\left(M_{i, j}^{(a)}\right)_{i, j \in[k]}$ the matrix of generalized events represented in Figure 4, that is, $M_{i, j}^{(a)}=M_{\beta, i, j}^{\prime} \equiv J_{i, j}^{f(i)}$ if $i \leqslant a, M_{i, j}^{(a)}=0$ if $i \in\{a+$ $1, \ldots, k-1\}$ and $j \neq i$, and $M_{i, i}^{(a)}=I_{i, i}^{f(i)}-J_{i, i}^{f(i)}$ if $i \in\{a+1, \ldots, k-1\}$, and $M_{k, j}^{(a)}=\Omega$. Note that $M^{(k-1)}=M_{\beta}^{\prime}$. For $a \in\{2,3, \ldots, k-1\}$, we also denote $N^{(a)}=\left(N_{i, j}^{(a)}\right)_{i, j \in[k]}$ the matrix of events which is the same as $M^{(a)}$ except the entry in position $(a, a)$ is $I_{a, a}^{f(a)}$ (see Figure 4).

By linearity of the $\mathbb{P}$-determinant with respect to matrix rows one gets for all $a \in$ $\{2,3, \ldots, k-1\}$,

$$
\mathbb{P} \operatorname{det}\left(M^{(a)}\right)=\mathbb{P} \operatorname{det}\left(N^{(a)}\right)+\mathbb{P} \operatorname{det}\left(M^{(a-1)}\right) .
$$

Thus, using this relation iteratively starting with $\mathbb{P} \operatorname{det}\left(M_{\beta}^{\prime}\right)=\mathbb{P} \operatorname{det}\left(M^{(k-1)}\right)$ we get

$$
\mathbb{P} \operatorname{det}\left(M_{\beta}^{\prime}\right)=\mathbb{P} \operatorname{det}\left(M^{(k-1)}\right)=\mathbb{P} \operatorname{det}\left(M^{(1)}\right)+\sum_{a=2}^{k-1} \mathbb{P} \operatorname{det}\left(N^{(a)}\right) .
$$

We will now prove that $\mathbb{P} \operatorname{det}\left(M^{(1)}\right)=(-1)^{k-1} \frac{\left|\mathcal{R}_{\mathbf{q}, r-1}\right|}{\left|\mathcal{S}_{\mathbf{p}, r}\right|}$, and $\mathbb{P} \operatorname{det}\left(N^{(a)}\right)=0$ for all $a \in$ $\{2,3, \ldots, k-1\}$.

Lemma 21. The $\mathbb{P}$-determinant of the matrix $M^{(1)}$ appearing in (14) is $\operatorname{Pdet}\left(M^{(1)}\right)=$ $(-1)^{k-1} \frac{\left|\mathcal{R}_{\boldsymbol{q}, r-1}\right|}{\left|\mathcal{S}_{p, r}\right|}$ 
Proof. This proof is similar to the one of Lemma 13. One easily gets

$$
\begin{aligned}
\mathbb{P} \operatorname{det}\left(M^{(1)}\right) & =\mathbb{P}\left(\bigcap_{i=2}^{k-1}\left(J_{i, i}^{f(i)}-I_{i, i}^{f(i)}\right) \cap\left(J_{1,1}^{f(1)}-J_{1, k}^{f(1)}\right)\right) \\
& =(-1)^{k-1} \mathbb{P}\left(\bigcap_{i=2}^{k-1}\left(I_{i, i}^{f(i)}-J_{i, i}^{f(i)}\right) \cap\left(J_{1, k}^{f(1)}-J_{1,1}^{f(1)}\right)\right) .
\end{aligned}
$$

Moreover, the generalized event inside the argument of $\mathbb{P}(\cdot)$ is clearly equivalent to the event " $S_{f(1)}=\{2,3, \ldots, k\}$ and for all $i \in\{2, \ldots, k-2\}, S_{f(i)}$ is a proper subset of $[k]$ ". This event is the same as " $S_{f(1)}=\{2,3, \ldots, k\}$ and $\mathbf{S} \in \mathcal{R}_{\mathbf{p}, r}$ " which has probability

$$
\mathbb{P}\left(\mathbf{S} \in \mathcal{R}_{\mathbf{p}, r}\right) \times \mathbb{P}\left(S_{f(1)}=\{2,3, \ldots, k\} \mid \mathbf{S} \in \mathcal{R}_{\mathbf{p}, r}\right)=\frac{\left|\mathcal{R}_{\mathbf{p}, r}\right|}{\left|\mathcal{S}_{\mathbf{p}, r}\right|} \times \frac{\left|\mathcal{R}_{\mathbf{q}, r-1}\right|}{\left|\mathcal{R}_{\mathbf{p}, r}\right|}=\frac{\left|\mathcal{R}_{\mathbf{q}, r-1}\right|}{\left|\mathcal{S}_{\mathbf{p}, r}\right|}
$$

It remains to prove that $\mathbb{P} \operatorname{det}\left(N^{(a)}\right)=0$ for all $a \in\{2,3, \ldots, k-1\}$. The proof is very similar to the one presented in Section 4.1 (indeed we point out that the rows $a-1$ and $a$ of $N^{(a)}$ are identical to the ones in Section 4.1). For $D \subseteq\{a+1, \ldots, k-1\}$, we denote by $N^{a, D}=\left(N_{i, j}^{a, D}\right)$ the matrix of generalized events defined by $N_{i, j}^{a, D}=N_{i, j}^{(a)}$ if $i \leqslant a$ or $j \neq i$, and $N_{i, i}^{a, D}=I_{i, i}^{f(i)}$ if $i \in D$, and $N_{i, i}^{a, D}=J_{i, i}^{f(i)}$ if $i \in\{a+1, \ldots, k-1\} \backslash D$. By linearity of the $\mathbb{P}$-determinant, one gets

$$
\mathbb{P} \operatorname{det}\left(N^{(a)}\right)=\sum_{D \subseteq\{a+1, \ldots, k-1\}}(-1)^{|D|} \mathbb{P} \operatorname{det}\left(N^{a, D}\right) .
$$

We now fix $a \in\{2,3, \ldots, k-1\}$ and $D \subseteq\{a+1, \ldots, k-1\}$ and proceed to prove that $\mathbb{P} \operatorname{det}\left(N^{a, D}\right)=0$. We define $\mathfrak{S}_{k}^{a}$ as the set of permutations $\pi$ of $[k]$ such that $\pi(i)=i$ for all $i>a$. We also define some subsets $K_{i, j}$ of $[k]$ by setting $K_{i, i}=[k]$ if $i \in D \cup\{a-1\}$, $K_{i, i}=\emptyset$ if $i \in\{a, a+1, \ldots, k-1\} \backslash D$, and $\left.\left.K_{i, j}=\right] i, j\right]$ for $i \in\{1,2, \ldots, a\}$, and $j \in[k] \backslash\{i\}$. By definition, a triple $(\mathbf{S}, f, \pi) \in \Omega_{k}$ is in $\Omega\left(N^{a, D}\right)$, if and only if the permutation $\pi$ is in $\mathfrak{S}_{k}^{a}$, and for all $i \in[k-1]$ the subset $K_{i, \pi(i)}$ is contained in $S_{f(i)}$. Lastly for $j \in[k-1]$ we denote

$$
H_{f, \pi, j}=\bigcup_{i \in[k-1] \backslash\{j\}, f(i)=f(j)} K_{i, \pi(i)} .
$$

We now define a partition of the set $\Omega\left(N^{a, D}\right)$, by declaring that a triple $(\mathbf{S}, f, \pi) \in \Omega\left(N^{a, D}\right)$ is in

- $\mathcal{W}$ if $f(a-1)=f(a)$,

- $\mathcal{X}$ if $f(a-1) \neq f(a), a \notin H_{f, \pi, a-1}$, and $a \notin H_{f, \pi, a}$,

- $\mathcal{Y}$ if $f(a-1) \neq f(a), a \notin H_{f, \pi, a-1}$, and $a \in H_{f, \pi, a}$,

- $\mathcal{Z}$ if $f(a-1) \neq f(a), a \in H_{f, \pi, a-1}$. 
Since $\Omega\left(N^{a, D}\right)=\mathcal{W} \uplus \mathcal{X} \uplus \mathcal{Y} \uplus \mathcal{Z}$, we get $\mathbb{P} \operatorname{det}\left(N^{a, D}\right)=\frac{W+X+Y+Z}{|\Omega|}$, where

$$
W=\sum_{(\mathbf{S}, f, \pi) \in \mathcal{W}} \epsilon(\pi), \quad X=\sum_{(\mathbf{S}, f, \pi) \in \mathcal{X}} \epsilon(\pi), \quad Y=\sum_{(\mathbf{S}, f, \pi) \in \mathcal{Y}} \epsilon(\pi), \quad \text { and } Z=\sum_{(\mathbf{S}, f, \pi) \in \mathcal{Z}} \epsilon(\pi) .
$$

We then show that $W=X=Y=Z=0$.

The proof that $W=0$ is identical to the proof of Lemma 16. The proof that $X=0$ is identical to the proof of Lemma 17. It is done by defining a sign reversing involution $\phi$ on $\mathcal{X}$. Explicitly, the mapping $\phi$ is defined on $\mathcal{X}$ by setting $\phi(\mathbf{S}, f, \pi)=\left(\mathbf{S}^{\prime}, f^{\prime}, \pi^{\prime}\right)$, where $f^{\prime}=f \circ(a-1, a)$, and $\pi^{\prime}=\pi \circ(a-1, a)$, and $\mathbf{S}^{\prime}$ is obtained from $\mathbf{S}$ simply by exchanging the presence or absence of the integer $a$ between the subsets $S_{f(a-1)}$ and $S_{f(a)}$. The proof that $\phi$ is an involution on $\mathcal{X}$ is identical to the one given in the proof of Lemma 17.

The proof that $Y=0$ is identical to the proof of Lemma 18. It is done by defining a sign reversing involution $\phi$ on $\mathcal{Y}$. Explicitly, one considers the partition $\mathcal{Y}=\mathcal{Y}_{1} \uplus \mathcal{Y}_{2} \uplus \mathcal{Y}_{3}$ defined exactly as in the proof of Lemma 18. One then defines a mapping $\phi$ on $\mathcal{Y}$ by setting $\phi(\mathbf{S}, f, \pi)=\left(\mathbf{S}, f, \pi^{\prime}\right)$, where $\pi^{\prime}=\pi \circ(a, k)$ (resp. $\left.\pi^{\prime}=\pi \circ(b, k), \pi^{\prime}=\pi \circ(a, b)\right)$ if $(\mathbf{S}, f, \pi)$ is in $\mathcal{Y}_{1}$ (resp. $\left.\mathcal{Y}_{2}, \mathcal{Y}_{3}\right)$, where $b$ is the greatest integer in $[k-1] \backslash\{a\}$ such that $f(b)=f(a)$ and $a \in K_{b, \pi(b)}$. The fact that $\phi$ is an involution on $\mathcal{Y}$ (actually, on each of the subsets $\mathcal{Y}_{1}, \mathcal{Y}_{2}$, and $\mathcal{Y}_{3}$ ) is identical to the one given in the proof of Lemma 18.

The proof that $Z=0$ is again identical to the proof of Lemma 19. This completes the proof that $\mathbb{P} \operatorname{det}\left(N^{a, D}\right)=0$ for all $a \in\{2,3, \ldots, k-1\}$ and all $D \subseteq\{a+1, \ldots, k-1\}$. This together with Lemma 21 complete the proof of Proposition 20, hence of the case $(b)$ of Theorem 1.

\subsection{Computing the $\mathbb{P}$-determinant of the matrix $M_{\gamma}$.}

In this section we compute the $\mathbb{P}$-determinant of the matrix $M_{\gamma}$.

Lemma 22. The $\mathbb{P}$-determinant of the matrix $M_{\gamma}$ defined in Proposition 10 is given by

$$
\operatorname{Pdet}\left(M_{\gamma}\right)=\sum_{a=1}^{k} \mathbb{P} \operatorname{det}\left(Q^{(a)}\right),
$$

where $Q^{(a)}=\left(Q_{i, j}^{(a)}\right)_{i, j \in[k]}$ is the matrix of generalized events defined by $Q_{i, j}^{(a)}=J_{i, j+1}^{f(i)}$ if $i \in[a-1], Q_{i, j}^{(a)}=J_{i, j+1}^{f(i-1)}$ if $i \in\{a+1, \ldots, k\}$, and $Q_{a, j}^{(a)}=\Omega$.

Note that $Q^{(k)}=M_{\beta}$, where $M_{\beta}$ is the matrix defined in Proposition 10 .

Corollary 23. The $\mathbb{P}$-determinant of the matrix $M_{\gamma}$ defined in Proposition 10 is

$$
\operatorname{Pdet}\left(M_{\gamma}\right)=\sum_{a=1}^{k} \frac{\left|\mathcal{R}_{\boldsymbol{q}^{(a)}, r-1}\right|}{\left|\mathcal{S}_{\boldsymbol{p}, r}\right|}
$$

where $\boldsymbol{q}^{(a)}=\left(q_{1}^{(a)}, \ldots, q_{k}^{(a)}\right)$ and for all $i \in[k], q_{i}^{(a)}=p_{i}-1$ if $i \neq j$ and $q_{a}^{(a)}=p_{a}$. 
Proof of Corollary 23. By Proposition 20,

$$
\operatorname{Pdet}\left(Q^{(k)}\right) \equiv \mathbb{P} \operatorname{det}\left(M_{\beta}\right)=\frac{\left|\mathcal{R}_{\mathbf{q}^{(1)}, r-1}\right|}{\left|\mathcal{S}_{\mathbf{p}, r}\right|} .
$$

Now for $a \in[k-1]$, permuting cyclically $k-a$ times both the rows and the columns of the matrix $Q^{(a)}$ gives

$$
\mathbb{P} \operatorname{det}\left(Q^{(a)}\right)=\mathbb{P} \operatorname{det}\left(R^{(a)}\right),
$$

where $R^{(a)}=\left(R_{i, j}^{(a)}\right)_{i, j \in[k]}$ is the matrix defined by $R_{i, j}^{(a)}=J_{i+a, j+a+1}^{f(i+a-1)}$ for $i \in[k-a]$, $R_{i, j}=J_{i+a, j+a+1}^{f(i+a-k)}$ for $i \in\{k-a+1, \ldots, k-1\}$, and $R_{k, j}^{(a)}=\Omega$. Since the surjection $f$ is uniformly random we get,

$$
\mathbb{P} \operatorname{det}\left(R^{(a)}\right)=\mathbb{P} \operatorname{det}\left(T^{(a)}\right),
$$

where $T^{(a)}=\left(T_{i, j}^{(a)}\right)_{i, j \in[k]}$ is the matrix defined by $T_{i, j}^{(a)}=J_{i+a, j+a+1}^{f(i)}$ for $i \in[k-1]$ and $T_{k, j}^{(a)}=\Omega$. Now, by symmetry (obtained by replacing the integer $i$ by $i+a$ in the subsets $\left.S_{1}, \ldots, S_{r}\right)$, the $\mathbb{P}$-determinant $\mathbb{P} \operatorname{det}\left(T^{(a)}\right)$ is obtained from $\mathbb{P} \operatorname{det}\left(M_{\beta}\right)$ by replacing $p_{i}$ by $p_{i+a}$ for all $i \in[k]$, that is,

$$
\operatorname{Pdet}\left(T^{(a)}\right)=\frac{\left|\mathcal{R}_{\mathbf{q}^{(a+1)}, r-1}\right|}{\left|\mathcal{S}_{\mathbf{p}, r}\right|}
$$

This together with Lemma 22 completes the proof of Corollary 23.

Note that Corollary 23 together with Proposition 10 prove the case $(c)$ of Theorem 1. It now only remains to prove Lemma 22. For $D \subseteq[k-1]$ and $j \in[k]$, we denote $M^{D}=\left(M_{i, j}^{D}\right)_{i, j \in[k]}$ the matrix of events defined by $M_{i, j}^{D}=J_{i-1, j+1}^{f(i)}$ if $i \in D, M_{i, j}^{D}=J_{i, j+1}^{f(i)}$ if $i \in[k-1] \backslash D$, and $M_{k, j}^{D}=\Omega$. By the multilinearity of $\mathbb{P}$-determinants with respect to the rows,

$$
\mathbb{P} \operatorname{det}\left(M_{\gamma}\right)=\sum_{D \subseteq[k-1]}(-1)^{|D|} \mathbb{P} \operatorname{det}\left(M^{D}\right) .
$$

We now show that only $k$ of the subsets $D$ contribute to the above sum.

Claim. If $D \subseteq[k-1]$ contains an integer $a>1$ but not $a-1$, then $\mathbb{P} \operatorname{det}\left(M^{D}\right)=0$.

Proof of the claim. We suppose that the subset $D$ contains the integer $a>1$ but not $a-1$. We remark that the $(a-1)$ th and ath rows of the matrix $M^{D}$ are almost identical: indeed for all $j \in[k], M_{a-1, j}^{D}=J_{a-1, j+1}^{f(a-1)}$ while $M_{a, j}^{D}=J_{a-1, j+1}^{f(a)}$. We now show that this implies $\mathbb{P} \operatorname{det}\left(M^{D}\right)=0$. We define a mapping $\phi$ on $\Omega\left(M^{D}\right)$ by setting $\phi(\mathbf{S}, f, \pi)=(\mathbf{S}, f \circ(a-1, a), \pi \circ(a-1, a))$. We want to prove that $\phi$ is an involution on $\Omega\left(M^{D}\right)$. Let $(\mathbf{S}, f, \pi) \in \Omega_{k}$. It is clear that $f \circ(a, a-1)$ is a surjection from $[k-1]$ to $[r]$ so that $\phi(\mathbf{S}, f, \pi)$ is in $\Omega_{k}$. Moreover, it is easy to see from the above remark about the $(a-1)$ th and ath rows of $M^{D}$ that $\phi(\mathbf{S}, f, \pi)$ is in $\Omega\left(M^{D}\right)$ (indeed the triple $\phi(\mathbf{S}, f, \pi)$ satisfies the condition $M_{a-1, \pi(a-1)}^{D}$ because $(\mathbf{S}, f, \pi)$ satisfies $M_{a, \pi(a)}^{D}$, and $\phi(\mathbf{S}, f, \pi)$ satisfies 
$M_{a, \pi(a)}^{D}$ because $(\mathbf{S}, f, \pi)$ satisfies $\left.M_{a-1, \pi(a-1)}^{D}\right)$. Thus the mapping $\phi$ is an involution of $\Omega\left(M^{D}\right)$. Moreover, since the involution $\phi$ changes the sign of the permutation $\pi$ we get

$$
\operatorname{Pdet}\left(M^{D}\right)=\frac{1}{|\Omega|} \sum_{(\mathbf{S}, f, \pi) \in \Omega\left(M^{D}\right)} \epsilon(\pi)=0,
$$

as claimed.

So far we have proved that

$$
\mathbb{P} \operatorname{det}\left(M_{\gamma}\right)=\mathbb{P} \operatorname{det}\left(M^{\emptyset}\right)+\sum_{a=1}^{k-1}(-1)^{a} \mathbb{P} \operatorname{det}\left(M^{[a]}\right) .
$$

Moreover, by definition $M^{\emptyset}=Q^{(k)}$, and it is easy to see, by reordering the rows of $M^{[a]}$, that for all $a \in[k-1]$ we have $\mathbb{P} \operatorname{det}\left(M^{[a]}\right)=(-1)^{a} \mathbb{P} \operatorname{det}\left(Q^{(a)}\right)$. This completes the proof of Lemma 22, hence of the case $(c)$ of Theorem 1.

\section{Open questions and conjectures}

This paper leaves open a few questions. First of all, the proof of Theorem 1 that we obtained is not as elegant as we had hoped for, hence the following question:

Question 24. Is there a more direct proof of Theorem 1? In particular, can one prove Theorem 1 without using the matrix-tree theorem?

Second, our use of the matrix-tree theorem (for which a "forest version" exists) suggests the following question:

Question 25. For $\zeta \in\{\alpha, \beta, \delta\}$, is there a simple expression for the probability that the random graph $G_{\zeta}(\boldsymbol{S}, f)$ is a pseudo-forest, that is, a digraph in which the only cycles are loops (so that the connected components are trees oriented toward a vertex which has a loop)?

More importantly, Theorem 1 gives simple formulas for the probability that certain random graphs are trees. We stumbled over one of these formulas while studying a factorization problem in the symmetric group [1], but it is unclear how general this phenomenon is.

Question 26. Is there a more general theory encompassing Theorem 1?

We conclude with a few conjectures about a random digraph $G_{\delta}$ defined analogously to $G_{\alpha}, G_{\beta}$, and $G_{\gamma}$. Let $\delta$ be the mapping taking as argument an integer $i \in[k]$ and a subset $S \subseteq[k]$ and defined by $\delta(i, S)=i$ if $i \in S$, and otherwise $\delta(i, S)$ is the integer $j \in[k] \backslash S$ such that $] i, j-1] \subseteq S$. The mapping $\delta$ is represented in Figure 5 .

Given a tuple $\mathbf{S}=\left(S_{1}, \ldots, S_{r}\right)$ in $\mathcal{S}_{\mathbf{p}, r}$ and a surjection $f$ from $[k-1]$ to $[r]$, we define $G_{\delta}(\mathbf{S}, f)$ to be the digraph with vertex set $[k]$ and arc set $A=\left\{a_{1}, \ldots, a_{k-1}\right\}$ where $a_{i}=\left(i, \delta\left(i, S_{f(i)}\right)\right)$ for all $i \in[k-1]$. The following conjecture suggests there is a simple expression for the probability that $G_{\delta}(\mathbf{S}, f)$ is a tree. 


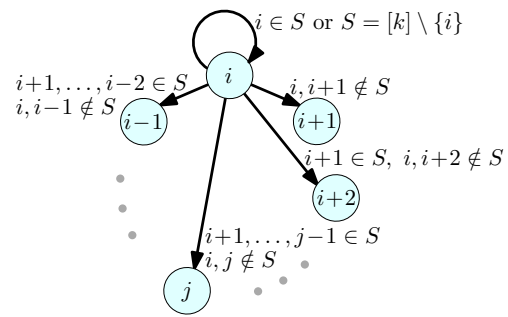

( $\delta$ ) Rule " $\bar{i}, i+1, \ldots, j-1, \bar{j} "$.

Figure 5: Rule $\delta$ for creating an arc of the complete graph $K_{k}$.

Conjecture 27. Let $k$ and $r$ be positive integers such that $r<k$, and let $\boldsymbol{p}=\left(p_{1}, \ldots, p_{k}\right)$ be a tuple of non-negative integers. Let $\boldsymbol{S}=\left(S_{1}, \ldots, S_{r}\right)$ be a uniformly random element of $\mathcal{R}_{p, r}$ (supposing that this set is non-empty), and let $f$ be a uniformly random surjection from $[k-1]$ to $[r]$ independent from $\boldsymbol{S}$. Then the probability $P_{\delta}(\boldsymbol{p}, r)$ that the random digraph $G_{\delta}(\boldsymbol{S}, f)$ is a tree is

$$
P_{\delta}(\boldsymbol{p}, r)=\frac{\left|\mathcal{R}_{p, r-1}\right|}{\left|\mathcal{R}_{p, r}\right|} .
$$

This is equal to the probability that $S_{1}=\emptyset$.

Although we were unable to prove Conjecture 27, we obtained the following analogue of Proposition 10:

$$
P_{\delta}(\mathbf{p}, r)=\frac{\left|\mathcal{S}_{\mathbf{p}, r}\right|}{\left|\mathcal{R}_{\mathbf{p}, r}\right|} \mathbb{P} \operatorname{det}\left(M_{\delta}\right)
$$

where $M_{\delta}=\left(M_{\delta, i, j}\right)_{i, j \in[k]}$ is the matrix of the generalized event defined by $M_{\delta, i, j}=I_{i, j}^{f(i)}$ $J_{i-1, j}^{f(i)}$ for $i \in[k-1]$, and $M_{\delta, k, j}=\Omega$. Thus Conjecture 27 is equivalent to the following identity:

$$
\mathbb{P} \operatorname{det}\left(M_{\delta}\right)=\frac{\left|\mathcal{R}_{\mathbf{p}, r-1}\right|}{\left|\mathcal{S}_{\mathbf{p}, r}\right|} .
$$

One approach to try to prove $(15)$ is to use the multilinearity of $\mathbb{P}$-determinants. For $D \subseteq[k-1]$ and $j \in[k]$, we denote $M^{D}=\left(M_{i, j}^{D}\right)_{i, j \in[k]}$, where $M_{i, j}^{D}=J_{i-1, j}^{f(i)}$ if $i \in D$, $M_{i, j}^{D}=I_{i, j}^{f(i)}$ if $i \in[k-1] \backslash D$, and $M_{k, j}^{D}=\Omega$. By multilinearity of $\mathbb{P}$-determinants,

$$
\mathbb{P} \operatorname{det}\left(M_{\delta}\right)=\sum_{D \subseteq[k-1]}(-1)^{|D|} \mathbb{P} \operatorname{det}\left(M^{D}\right) .
$$

Calculations suggest the following formula for the $\mathbb{P}$-determinant of $M^{D}$ :

Conjecture 28. For all $D \subseteq[k-1]$,

$$
\mathbb{P} \operatorname{det}\left(M^{D}\right)=\frac{\left|\mathcal{R}_{p, r}\right|}{\left|\mathcal{S}_{p, r}\right|} \times \mathbb{P}\left(k \notin S_{1}, D \subseteq S_{1} \mid \boldsymbol{S} \in \mathcal{R}_{\boldsymbol{p}, r}\right)
$$


Remark 29. Conjecture 28 implies Conjecture 27 since substituting the conjectured formula for $\mathbb{P} \operatorname{det}\left(M^{D}\right)$ in (16) and doing inclusion-exclusion gives

$$
\mathbb{P}\left(M_{\delta}\right)=\frac{\left|\mathcal{R}_{\mathbf{p}, r}\right|}{\left|\mathcal{S}_{\mathbf{p}, r}\right|} \times \mathbb{P}\left(S_{1}=\emptyset \mid \mathbf{S} \in \mathcal{R}_{\mathbf{p}, r}\right) .
$$

Remark 30. When $D=\emptyset$ then $M^{D}=M_{\alpha}$ as defined in Proposition 10. Hence, in this case Conjecture 28 holds by Proposition 12. Similarly, when $D=[k-1]$ the matrix $M^{D}$ is the matrix $M_{\beta}$ as defined in Proposition 10, up to shifting cyclically the $k$ columns one position to the right and shifting the first $k-1$ rows one position down. Hence, in this case Conjecture 28 holds by Proposition 20.

\section{Acknowledgements}

We thank Omer Angel, Robin Pemantle, and Peter Winkler for interesting discussions. We thank an anonymous referee for pointing out that Lemma 7 is a direct consequence of the classical matrix-tree theorem over arbitrary commutative rings.

\section{References}

[1] O. Bernardi and A.H. Morales. Bijections and symmetries for the factorizations of the long cycle. Adv. in Appl. Math., 50:702-722, 2013, doi:10.1016/j. aam.2013.01.004.

[2] D. M. Jackson. Some combinatorial problems associated with products of conjugacy classes of the symmetric group. J. Combin. Theory Ser. A, 49:363-369, 1988, doi : 10.1016/0097-3165(88)90062-3.

[3] R. Kenyon and P. Winkler. Branched polymers. Amer. Math. Monthly, 7:612-628, 2009, doi:10.4169/193009709X458582.

[4] D. Zeilberger. A combinatorial approach to matrix algebra. Discrete Math., 56:61-72, 1985, doi : 10.1016/0012-365X (85)90192-X. 